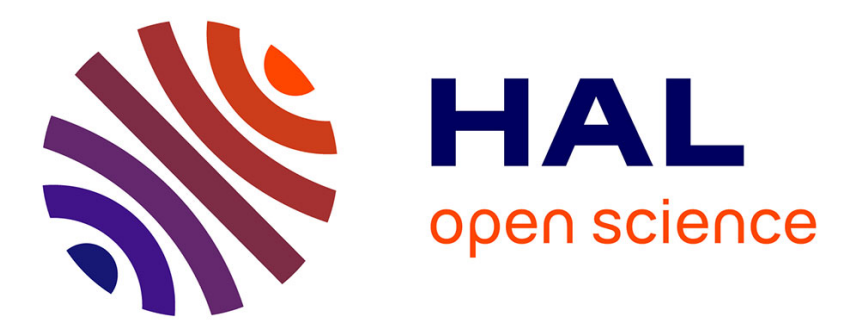

\title{
Anisotropic linear forcing for synthetic turbulence generation in large eddy simulation and hybrid RANS/LES modeling
}

\author{
Benoît de Laage de Meux, B Audebert, Remi Manceau, R. Perrin
}

\section{To cite this version:}

Benoît de Laage de Meux, B Audebert, Remi Manceau, R. Perrin. Anisotropic linear forcing for synthetic turbulence generation in large eddy simulation and hybrid RANS/LES modeling. Physics of Fluids, 2015, 27, pp.35. 10.1063/1.4916019 hal-01246100

\section{HAL Id: hal-01246100 \\ https://inria.hal.science/hal-01246100}

Submitted on 18 Dec 2015

HAL is a multi-disciplinary open access archive for the deposit and dissemination of scientific research documents, whether they are published or not. The documents may come from teaching and research institutions in France or abroad, or from public or private research centers.
L'archive ouverte pluridisciplinaire HAL, est destinée au dépôt et à la diffusion de documents scientifiques de niveau recherche, publiés ou non, émanant des établissements d'enseignement et de recherche français ou étrangers, des laboratoires publics ou privés. 


\title{
Anisotropic linear forcing for synthetic turbulence generation in large eddy simulation and hybrid RANS/LES modeling
}

\author{
B. de Laage de Meux, ${ }^{1, a)}$ B. Audebert, ${ }^{1}$ R. Manceau, ${ }^{2,3}$ and R. Perrin ${ }^{2}$ \\ ${ }^{1}$ EDF RED D, Fluid Mechanics, Energy and Environment Department, 6 quai Watier, \\ 78401 Chatou, France \\ ${ }^{2}$ Department of Fluid Flow, Heat Transfer and Combustion, Institute Pprime, \\ CNRS-University of Poitiers-ENSMA, SP2MI, Blvd. Marie et Pierre Curie, BP 30179, \\ 86962 Futuroscope Chasseneuil Cedex, France \\ ${ }^{3}$ Department of Applied Mathematics, CNRS-University of Pau and Inria CAGIRE group, \\ Avenue de l'université, 64013 Pau, France
}

(Received 22 September 2014; accepted 11 March 2015; published online 27 March 2015)

\begin{abstract}
A general forcing method for Large Eddy Simulation (LES) is proposed for the purpose of providing the flow with fluctuations that satisfy a desired statistical state. This method, the Anisotropic Linear Forcing (ALF) introduces an unsteady linear tensor function of the resolved velocity which acts as a restoring force in the mean velocity and resolved stress budgets. The ALF generalizes and extends several forcing previously proposed in the literature. In order to make it possible to impose the integral length scale of the turbulence generated by the forcing term, an alternative formulation of the ALF, using a differential spatial filter, is proposed and analyzed. The anisotropic forcing of the Reynolds stresses is particularly attractive, since unsteady turbulent fluctuations can be locally enhanced or damped, depending on the target stresses. As such, it is shown that the ALF is an effective method to promote turbulent fluctuations downstream of the LES inlet or at the interface between RANS and LES in zonal hybrid RANS/LES modeling. The detailed analysis of the influence of the ALF parameters in spatially developing channel flows and hybrid computations where the ALF target statistics are given by a RANS second-moment closure show that this original approach performs as well as the synthetic eddy method. However, since the ALF method is more flexible and significant computational savings are obtained, the method appears a promising all-in-one solution for general embedded LES simulations. (C) 2015 AIP Publishing LLC. [http://dx.doi.org/10.1063/1.4916019]
\end{abstract}

\section{INTRODUCTION}

In many industrial applications, the unsteady description of large scale, energetic structures of turbulence is of major interest in order to predict unsteady aerodynamics loads and thermal fatigue, among others. While Large Eddy Simulation (LES) is able to capture these structures, the prohibitive numerical cost in the near-wall region hinders the spreading of this method into the daily engineering practice. Therefore, for industrial studies of high Reynolds number wall-bounded flows, the use of LES will be restricted to specific regions of the flow, for the foreseeable future. In response, an intense research effort is nowadays dedicated to the development of hybrid RANS/LES modeling of turbulence, offering an intermediate resolution between LES and a low-cost statistical description of the flow (RANS). In many of these new generation approaches, one single set of model equations is used in the whole domain and the level of description of turbulence is locally selected based on grid spacing and/or flow conditions, resulting in RANS in the near-wall region and LES in the core region (see Refs. 1-3, for instance). These approaches are referred

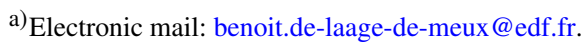


to as global hybrid RANS/LES methods in the classification of Sagaut et al. ${ }^{4}$ A second class of methods, referred to as zonal hybrid RANS/LES methods, restricts the RANS and LES models to separated sub-domains and aims at properly interface the two models. This approach is well suited for applications where the unsteady description of the flow is only required in a reduced region of interest, whereas the influence of the surrounding environment on this region demands a much larger computational domain. For instance, such situations arise in the numerical study of the sealing systems of hydraulic turbomachinery: on the one hand, an unsteady resolution of turbulence using LES is mandatory in some specific low Reynolds number regions of mixing of hot and cold water; on the other hand, the computational domain must be extended by a far larger RANS region, in order to take into account several other physical phenomena such as rotor/stator interactions and heat exchanges.

One of the main challenges for hybrid methods consists in correctly matching the LES and RANS variables at the interface, be it discontinuous (zonal approach) or diffuse (global approach). When the RANS domain is upstream of the LES domain, referred to as RANS to LES coupling below, the problem is closely related to the problem of inflow boundary conditions for spatially developing LES. The objective is to enrich the RANS solution, representative of those of a developed flow, in order to reduce the development distance downstream of the beginning of the LES region. A comparative study of several methods of generation of such inflow conditions was provided by Keating et al. ${ }^{5}$ The parameters of the method must be fully determined by the RANS and the LES computations. A few applications on relatively simple geometries are reported in the literature. ${ }^{6,7}$ It is worth noting that synthetic turbulence methods, ${ }^{8,9}$ for which the turbulent fluctuations are directly modeled, appear easier to parametrize from the local low order statistics of RANS than more advanced methods, based on recycling procedures, ${ }^{10}$ for instance.

In association with generation of synthetic turbulent fluctuations at the interface, a second coupling approach investigated in the literature is a volume forcing of the momentum equation. A few authors ${ }^{11}$ have considered a feedback forcing in the RANS equations. Various forcing methods have been proposed for various RANS/LES configurations. Spille-Kohoff and Kaltenbach ${ }^{12}$ (SKK) proposed a fluctuating forcing of the wall-normal velocity in order to promote turbulent fluctuations in a set of forcing planes close to the LES inlet. Their forcing is based on a controller based on the turbulent shear stress. This technique has been successfully applied by Keating et al., ${ }^{6}$ in association with a synthetic turbulence generation method. Laraufie et al. ${ }^{13}$ (LDS) proposed several modifications of this method. In particular, the controller is based on the wall normal turbulent stress rather than the shear stress. They studied in detail the influence of different parameters of the method and showed that, associated with the synthetic eddy method ${ }^{9}$ to generate fluctuating boundary conditions at the inlet, using the refined version proposed by Pamiès et al. ${ }^{14}$ the forcing yields an appreciable reduction of the adaptation distance downstream of the inlet. A volume forcing of the LES has also been proposed by Schlüter et al. ${ }^{15}$ in the context of LES to RANS zonal coupling, i.e., when the RANS domain is downstream of the LES domain. This forcing of the mean flow was applied in order to sensitize the upstream LES to the downstream RANS computation despite uncoupled outflow boundary conditions for the LES, in order to avoid reflections. ${ }^{16}$ Note that Benarafa et al. ${ }^{17}$ have employed the forcing of Schlüter et al. ${ }^{15}$ in the whole LES domain, in order to achieve unsteady computations with realistic velocity moments, despite a relatively coarse mesh. A comparable hybridization of the resolution is the NLDE approach. ${ }^{18}$ Similarly, Xiao and Jenny ${ }^{11}$ have recently proposed a two-way forcing of entirely overlapping RANS and LES domains for the purpose of obtaining a consistent formalism for dual-mesh hybrid RANS/LES modeling. Although their forcing in the LES momentum equation has the potential to individually control all the components of the resolved stress tensor, only the resolved kinetic energy is actually controlled in the applications presented in their study.

In the present paper, a new body force is proposed, in order to impose, in a restricted zone at the beginning of the LES region, target statistics of the flow (mean velocities and resolved stresses). Although this forcing can potentially be extended to global hybrid RANS/LES methods, the present article focuses on zonal coupling. The method is derived based on the analysis of the statistical effects of momentum forcing, which is described in Sec. II A. After a brief discussion, within this statistical framework, of the various forcings proposed in the literature (Sec. II B), the present 
method, the so-called Anisotropic Linear Forcing (ALF), is derived (Sec. II C). The main characteristic of the ALF is that the force linearly depends on the instantaneous velocity via a tensorial relation. Therefore, it is shown in Sec. II D that it actually generalizes the linear isotropic forcing introduced in the context of isotropic turbulence by Lundgren ${ }^{19}$ and further analyzed by Rosales and Meneveau. ${ }^{20}$ Section III is dedicated to the validation of the ALF in homogeneous turbulence. The case of isotropic turbulence is considered first, in order to verify that the interesting properties of the isotropic linear forcing exhibited by Rosales and Meneveau ${ }^{20}$ are recovered with the present formulation. Then the ALF is applied in various anisotropic homogeneous cases, aiming to demonstrate that it is able to force the LES towards any anisotropic turbulent state. In Sec. IV, the more complex case of spatially developing channel flow is considered. The ALF is first applied to the whole computation domain of a LES forced towards its fully developed moments. This case is used to discuss the influence of the parameters of the forcing. Applications of the ALF to zonal RANS to LES coupling are presented in Sec. IV B. The forcing is applied in a reduced area downstream the LES inlet and overlapping the upstream RANS region, in order to generate turbulent fluctuations in the LES domain, and compared with the synthetic eddy method at the LES inlet.

\section{MATHEMATICAL FRAMEWORK}

\section{A. Statistical effect of a forcing}

The filtered momentum equation of LES for an incompressible flow with an additional body force $f_{i}$ is considered,

$$
\frac{\partial \widetilde{u_{i}}}{\partial t}+\widetilde{u_{j}} \frac{\partial \widetilde{u_{i}}}{\partial x_{j}}=-\frac{1}{\rho} \frac{\partial \widetilde{p}}{\partial x_{i}}+v \frac{\partial^{2} \widetilde{u_{i}}}{\partial x_{j} \partial x_{j}}-\frac{\partial \tau_{i j}}{\partial x_{j}}+f_{i},
$$

where the tilde $\widetilde{r}$ denotes the LES filtering operator and $\tau_{i j}=\widetilde{u_{i} u_{j}}-\widetilde{u_{i}} \widetilde{u_{j}}$ is the subgrid scale tensor. The body force $f_{i}$ can be split into its Reynolds averaged part $\left\langle f_{i}\right\rangle$ and its fluctuating part $f_{i}^{\prime}$ as

$$
f_{i}=\left\langle f_{i}\right\rangle+f_{i}^{\prime} .
$$

Using this decomposition, the contribution of $f_{i}$ to the mean filtered momentum equation writes (throughout this paper, angular brackets $\langle\cdot\rangle$ are used to indicate a statistical average)

$$
\frac{\partial\left\langle\widetilde{u_{i}}\right\rangle}{\partial t}+\frac{\partial}{\partial x_{j}}\left(\left\langle\widetilde{u_{i}}\right\rangle\left\langle\widetilde{u_{j}}\right\rangle\right)=-\frac{1}{\rho} \frac{\partial\langle\widetilde{p}\rangle}{\partial x_{i}}+v \frac{\partial^{2}\left\langle\widetilde{u_{i}}\right\rangle}{\partial x_{j}^{2}}-\frac{\partial}{\partial x_{j}}\left\langle u_{i}^{\prime} u_{j}^{\prime}\right\rangle-\frac{\partial\left\langle\tau_{i j}\right\rangle}{\partial x_{j}}+\left\langle f_{i}\right\rangle,
$$

where

$$
u_{i}^{\prime}=\widetilde{u_{i}}-\left\langle\widetilde{u_{i}}\right\rangle,
$$

such that $\left\langle u_{i}^{\prime} u_{j}^{\prime}\right\rangle$ is the resolved part of the Reynolds stress tensor. Obviously, only the averaged part $\left\langle f_{i}\right\rangle$ of the body force acts on the mean flow. It is also useful to exhibit the contribution of $f_{i}$ in the equation for the resolved stress. Eqs. (1) and (3) lead to

$$
\frac{\partial\left\langle u_{i}^{\prime} u_{j}^{\prime}\right\rangle}{\partial t}+\left\langle\widetilde{u_{j}}\right\rangle \frac{\partial\left\langle u_{i}^{\prime} u_{j}^{\prime}\right\rangle}{\partial x_{j}}=P_{i j}^{r}+P_{i j}^{f}+\phi_{i j}^{r}+\chi_{i j}+D_{i j}^{T r}+D_{i j}^{\tau}+D_{i j}^{v r}-\varepsilon_{i j}^{r},
$$

where $P_{i j}^{r}, \phi_{i j}^{r}, D_{i j}^{T r}, D_{i j}^{v r}$, and $\varepsilon_{i j}^{r}$ denote the resolved production, pressure-strain, turbulent diffusion, viscous diffusion, and dissipation terms, analogous to those involved in the unfiltered Reynolds stress equations, and

$$
\begin{aligned}
& D_{i j}^{\tau}=-\frac{\partial}{\partial x_{l}}\left\langle\tau_{i l}^{\prime} u_{j}^{\prime}+\tau_{j l}^{\prime} u_{i}^{\prime}\right\rangle, \\
& \chi_{i j}=\left\langle\tau_{i l}^{\prime} \frac{\partial u_{j}^{\prime}}{\partial x_{l}}+\tau_{j l}^{\prime} \frac{\partial u_{i}^{\prime}}{\partial x_{l}}\right\rangle .
\end{aligned}
$$

The forcing introduces the additional term

$$
P_{i j}^{f}=\left\langle f_{i}^{\prime} u_{j}^{\prime}\right\rangle+\left\langle f_{j}^{\prime} u_{i}^{\prime}\right\rangle
$$


that only depends on the fluctuating part of the force. This term increases or damps the resolved stresses, depending on the sign. In Sec. II B, the action of the forcing is analyzed through these two aspects: its effect on the mean flow (Eq. (3)) and its effect on the resolved stresses (Eq. (7)).

\section{B. Analysis of previously proposed forcing methods}

\section{Forcing of the mean flow}

The mathematical formulation of the forcing introduced by Schlüter et al. ${ }^{15}$ is

$$
f_{i}=\frac{1}{\tau_{f}}\left(\left\langle\widetilde{u}_{i}\right\rangle^{\dagger}-\left\langle\widetilde{u}_{i}\right\rangle\right),
$$

where $\tau_{f}$ is a time scale and $\left\langle u_{i}\right\rangle^{\dagger}$ is the target mean velocity. In order to estimate the mean filtered velocity $\left\langle\widetilde{u}_{i}\right\rangle$, in the framework of stationary flows, an explicit time filtering is applied, ${ }^{23}$ most commonly using an exponential filter, denoted by $\mathcal{G}_{T}$. In differential form, $\mathcal{G}_{T}$ is written as

$$
\frac{\partial \mathcal{G}_{T}(\phi)}{\partial t}=\frac{\phi-\mathcal{G}_{T}(\phi)}{T},
$$

where the parameter $T$ specifies the temporal width of the filter. Therefore, the forcing applied by Schlüter et al. can be written as

$$
f_{i}=\frac{1}{\tau_{f}}\left(\left\langle\widetilde{u_{i}}\right\rangle^{\dagger}-\mathcal{G}_{T}\left(\widetilde{u_{i}}\right)\right) .
$$

For stationary flows, $\mathcal{G}_{T}(\phi)$ goes to the statistical average $\langle\phi\rangle$ in the limit of infinite temporal filter widths, such that Eq. (8) is recovered. In this case, the forcing simply gives rise to

$$
\left\langle f_{i}\right\rangle=\frac{1}{\tau_{f}}\left(\left\langle{\widetilde{u_{i}}}^{\dagger}-\left\langle\widetilde{u_{i}}\right\rangle\right) \text { and } P_{i j}^{f}=0\right.
$$

in the mean momentum equations and the resolved stress budgets, respectively. As expected, the body force drives the mean filtered velocity $\left\langle\widetilde{u}_{i}\right\rangle$ towards its target RANS value $\left\langle\widetilde{u}_{i}\right\rangle^{\dagger}$. In contrast, the forcing has no direct effect on the resolved Reynolds stresses, which is consistent with the objective of Schlüter et al. ${ }^{15}$ and Benarafa et al.${ }^{17}$ to compel a developed LES to satisfy a particular mean flow field.

It is also instructive to investigate the behavior of Eq. (10) in the limit $T \rightarrow 0$. In this case, the Gaussian filtering reduces to $\mathcal{G}_{0}(\phi)=\phi$ and the forcing becomes

$$
f_{i}=\frac{1}{\tau_{f}}\left(\left\langle\widetilde{u_{i}}\right\rangle^{\dagger}-\widetilde{u_{i}}\right) .
$$

Although it is counter-intuitive, since the RANS and LES variables represent very different physical quantities, this forcing, also considered by Schlüter et al. ${ }^{15}$ in their parametric study, leads to

$$
\left\langle f_{i}\right\rangle=\frac{1}{\tau_{f}}\left(\left\langle\widetilde{u_{i}}\right\rangle^{\dagger}-\left\langle\widetilde{u_{i}}\right\rangle\right) \quad \text { and } \quad P_{i j}^{f}=-\frac{2}{\tau_{f}}\left\langle u_{i}^{\prime} u_{j}^{\prime}\right\rangle .
$$

Thus, this forcing tends to impose the target mean velocity, and at the same time, it acts as a destruction term for the resolved stresses. The fact that the forcing of Eq. (12) severely damps the turbulent fluctuations was reported by Schlüter et al. ${ }^{15}$

For the intermediate cases corresponding to $0<T<\infty$, the role played by the forcing can be clarified by considering its effect on the low-pass filtered stress,

$$
\left\langle\left(\mathcal{G}_{T}\left(\widetilde{u_{i}}\right)-\left\langle\widetilde{u_{i}}\right\rangle\right)\left(\mathcal{G}_{T}\left(\widetilde{u_{j}}\right)-\left\langle\widetilde{u_{j}}\right\rangle\right)\right\rangle .
$$

In the budget of this quantity, forcing (10) introduces the destruction term

$$
-\frac{2}{\tau_{f}}\left\langle\left(G_{T}\left(\widetilde{u_{i}}\right)-\left\langle\widetilde{u_{i}}\right\rangle\right)\left(G_{T}\left(\widetilde{u_{j}}\right)-\left\langle\widetilde{u_{j}}\right\rangle\right)\right\rangle,
$$

which damps turbulent fluctuations at time scales larger than the temporal filter width $T$. 


\section{Forcing of the fluctuations}

Two similar forcing methods were proposed by SKK ${ }^{12}$ and LDS. ${ }^{13}$

In order to enhance or damp local flow events that contribute to the production of the shear stress, ${ }^{5}$ the SKK forcing term acts in the wall normal direction only,

$$
f_{i}=r_{\mathrm{SKK}} u^{\prime} \delta_{i 2} \text {. }
$$

$r_{\mathrm{SKK}}$ is a factor determined by a Proportional-Integral (PI) controller defined as

$$
r_{\mathrm{SKK}}=\alpha e+\beta \int_{0}^{t} e \mathrm{~d} t^{\prime}, \quad \text { with } \quad e=\left\langle u^{\prime} v^{\prime}\right\rangle^{\dagger}-\left\langle u^{\prime} v^{\prime}\right\rangle
$$

where $\left\langle u^{\prime} v^{\prime}\right\rangle^{\dagger}$ is the target shear stress, given by a RANS computation. Note that the energy content of the subgrid scales is usually considered negligible, such that the Reynolds stresses computed by a RANS model are used, without correction, as the target for the LES resolved stresses. However, a wiser approach, using an estimate of the subgrid scale energy, is possible, as will be presented in Sec. IV B 1 . The parameters of the controller, $\alpha$ and $\beta$, are chosen in order to optimize the reduction of the error $e$. Finally, the forcing is only applied in flow regions where the following conditions are satisfied:

$$
\left|u^{\prime}\right|<0.6 U_{b}, \quad\left|v^{\prime}\right|<0.4 U_{b}, \quad\left|u^{\prime} v^{\prime}\right|<0.0015 U_{b}^{2} .
$$

Considering Eq. (16), it is immediately observed that $\left\langle f_{i}\right\rangle=0$. The SKK forcing has no direct effect on the mean flow, only on turbulent fluctuations, as was intended by the authors. The resolved stress production of the forcing is

$$
P_{11}^{f}=P_{23}^{f}=P_{33}^{f}=0, \quad P_{22}^{f}=2 r_{\mathrm{SKK}}\left\langle u^{\prime} v^{\prime}\right\rangle, \quad P_{12}^{f}=r_{\mathrm{SKK}}\left\langle u^{\prime 2}\right\rangle, \quad P_{23}^{f}=r_{\mathrm{SKK}}\left\langle u^{\prime} w^{\prime}\right\rangle .
$$

Since $\left\langle u^{\prime 2}\right\rangle>0$, the shear stress production $P_{12}^{f}$ due to the forcing is positive (resp. negative) when $\left\langle u^{\prime} v^{\prime}\right\rangle$ is smaller (resp. larger) than the target value $\left\langle u^{\prime} v^{\prime}\right\rangle^{\dagger}$, tending to adjust the resolved shear stress to the target. Therefore, from the statistical point of view, it can be seen that, by promoting wall-normal fluctuations, the forcing selectively produces resolved stresses $\left\langle v^{\prime 2}\right\rangle,\left\langle u^{\prime} v^{\prime}\right\rangle$ and $\left\langle v^{\prime} w^{\prime}\right\rangle$, at a rate depending on the gap between the resolved and target shear-stress $\left\langle u^{\prime} v^{\prime}\right\rangle$. When the target is approached, the control factor $r_{\mathrm{SKK}}$ goes to zero, such a way that the forcing term vanishes.

Similarly to the SKK forcing, the LDS forcing introduces a fluctuating force in the direction normal to the wall, but now proportional to the wall-normal, rather than streamwise, fluctuating velocity,

$$
f_{i}=r_{\mathrm{LDS}} v^{\prime} \delta_{i 2} \text {. }
$$

The LDS term yields $\left\langle f_{i}\right\rangle=0$, similar to the SKK forcing, but the turbulent production tensor is

$$
P_{11}^{f}=P_{13}^{f}=P_{33}^{f}=0, \quad P_{22}^{f}=2 r_{\mathrm{LDS}}\left\langle v^{\prime 2}\right\rangle, \quad P_{12}^{f}=r_{\mathrm{LDS}}\left\langle u^{\prime} v^{\prime}\right\rangle, \quad P_{23}^{f}=r_{\mathrm{LDS}}\left\langle v^{\prime} w^{\prime}\right\rangle .
$$

Moreover, the control factor $r_{\mathrm{LDS}}$ is evaluated from the wall-normal turbulent stress as

$$
r_{\mathrm{LDS}}=\alpha\left(\left\langle v^{\prime 2}\right\rangle^{\dagger}-\left\langle v^{\prime 2}\right\rangle\right),
$$

such that the $P_{22}^{f}$ component adjusts the wall normal turbulent stress to its target value.

Equations (18) and (20) reveal that, as a side effect, the SKK and LDS terms both introduce additional production of components other than those used in the control factor (i.e., $\left\langle v^{2}\right\rangle$ and $\left\langle v^{\prime} w^{\prime}\right\rangle$ for SKK, $\left\langle u^{\prime} v^{\prime}\right\rangle$ and $\left\langle v^{\prime} w^{\prime}\right\rangle$ for LDS). Since the controller focuses on a single resolved stress component, these forcing methods cannot control the anisotropy of the turbulence they generate, in contrast with the ALF method introduced in Sec. II C.

\section{Formulation of the ALF}

The anisotropic linear forcing takes the general form of a tensorial linear function

$$
f_{i}=A_{i j} \widetilde{u_{j}}+B_{i},
$$


where the deterministic parameters of the forcings $A_{i j}$ and $B_{i}$ are a second order tensor and a vector, respectively. Note that the forcing methods presented above, Eqs. (12), (16) and (19), all belong to this general type of forcing, with

$$
\begin{gathered}
A_{i j}=-\frac{1}{\tau_{f}} \delta_{i j}, \quad B_{i}=\frac{1}{\tau_{f}}\left\langle\widetilde{u}_{i}\right\rangle^{\dagger} ; \\
A_{i j}=r_{\mathrm{SKK}} \delta_{i 2} \delta_{j 2}, \quad B_{i}=-r_{\mathrm{SKK}}\langle\widetilde{v}\rangle \delta_{i 2} ;
\end{gathered}
$$

and

$$
A_{i j}=r_{\mathrm{LDS}} \delta_{i 2} \delta_{j 1}, \quad B_{i}=-r_{\mathrm{LDS}}\langle\widetilde{u}\rangle \delta_{i 2}
$$

respectively. For the sake of simplicity, and because it is sufficient for our purpose, the analysis below is restricted to the case of symmetric $A_{i j}$ tensors.

The mean part of $f_{i}$, which contributes to the mean momentum equation, is

$$
\left\langle f_{i}\right\rangle=A_{i j}\left\langle\widetilde{u_{j}}\right\rangle+B_{i},
$$

and the fluctuating part

$$
f_{i}^{\prime}=A_{i j} u_{j}^{\prime} \text {. }
$$

As a result, the body force of Eq. (22) contributes to the budget of the resolved stress $\left\langle u_{i}^{\prime} u_{j}^{\prime}\right\rangle$ through the production term (see Eq. (7))

$$
P_{i j}^{f}=A_{i k}\left\langle u_{j}^{\prime} u_{k}^{\prime}\right\rangle+A_{j k}\left\langle u_{i}^{\prime} u_{k}^{\prime}\right\rangle
$$

Therefore, the ALF bears some similarity to the natural process of turbulent production in Eq. (5),

$$
P_{i j}^{r}=-\frac{\partial\left\langle\widetilde{u_{i}}\right\rangle}{\partial x_{k}}\left\langle u_{j}^{\prime} u_{k}^{\prime}\right\rangle-\frac{\partial\left\langle\tilde{u}_{j}\right\rangle}{\partial x_{k}}\left\langle u_{i}^{\prime} u_{k}^{\prime}\right\rangle
$$

It is worth pointing out that turbulent production $P_{i j}^{r}$ arises from the inertial terms in the subgrid scale momentum equation, of the form $\left(\partial\left\langle\widetilde{u}_{j}\right\rangle / \partial x_{k}\right) u_{i}^{\prime}$, i.e., terms formally and dimensionally similar to fluctuating part (27) of the ALF, in which $A_{i j}$ plays the role of the mean velocity gradient. As a consequence, any tensor field $A_{i j}$ that satisfies Galilean invariance is physically admissible. Appendix A provides a formal proof, for the particular form of $A_{i j}$ and $B_{i}$ used below, that the force $f_{i}$ is objective, i.e., invariant under Euclidean transformations.

In order to drive the mean velocity $\left\langle\tilde{u}_{j}\right\rangle$ and the resolved stress tensor $\left\langle u_{i}^{\prime} u_{j}^{\prime}\right\rangle$ toward target values, the constraints

$$
\begin{gathered}
\left\langle f_{i}\right\rangle=\frac{1}{\tau_{v}}\left(\left\langle\widetilde{u_{i}}\right\rangle^{\dagger}-\left\langle\widetilde{u_{j}}\right\rangle\right), \\
P_{i j}^{f}=\frac{1}{\tau_{r}}\left(\left\langle u_{i}^{\prime} u_{j}^{\prime}\right\rangle^{\dagger}-\left\langle u_{i}^{\prime} u_{j}^{\prime}\right\rangle\right)
\end{gathered}
$$

are imposed, which act as restoring forces, with relaxation times $\tau_{v}$ and $\tau_{r}$ for the mean velocity and the resolved stress tensor, respectively. In the context of hybrid RANS/LES, $\left\langle\widetilde{u}_{i}\right\rangle^{\dagger}$ and $\left\langle u_{i}^{\prime} u_{j}^{\prime}\right\rangle^{\dagger}$ will be obtained from RANS variables (see Sec. IV B 1), but in general, target statistics of the forcing can be given, for instance, by an experiment, a DNS or a highly resolved LES. Eqs. (26), (30), (28), and (31) lead to the system

$$
\begin{gathered}
A_{i j}\left\langle\widetilde{u}_{j}\right\rangle+B_{i}=\frac{1}{\tau_{v}}\left(\left\langle{\widetilde{u_{i}}}^{\dagger}-\left\langle\widetilde{u_{j}}\right\rangle\right),\right. \\
A_{i k}\left\langle u_{j}^{\prime} u_{k}^{\prime}\right\rangle+A_{j k}\left\langle u_{i}^{\prime} u_{k}^{\prime}\right\rangle=\frac{1}{\tau_{r}}\left(\left\langle u_{i}^{\prime} u_{j}^{\prime}\right\rangle^{\dagger}-\left\langle u_{i}^{\prime} u_{j}^{\prime}\right\rangle\right),
\end{gathered}
$$

which implicitly determines the coefficients $A_{i j}$ and $B_{i}$ of the ALF. The statistics $\left\langle\widetilde{u}_{j}\right\rangle$ and $\left\langle u_{i}^{\prime} u_{j}^{\prime}\right\rangle$ are estimated during the computation. Eq. (33) is a second order tensorial algebraic equation, in the form,

$$
\mathbf{A R}+\mathbf{R A}=\mathbf{H} .
$$


This tensorial equation is frequently encountered in continuum mechanics and its analytical inversion has been extensively studied (Refs. 24 and 25 for instance). Hoger and Carlson ${ }^{26}$ provided a general solution under the form ${ }^{24}$

$$
\begin{aligned}
\mathbf{A}=\frac{1}{2}\left[I_{3}\left(I_{1} I_{2}-I_{3}\right)\right]^{-1} & {\left[I_{1} \mathbf{R}^{2} \mathbf{H} \mathbf{R}^{2}-I_{1}^{2}\left(\mathbf{R}^{2} \mathbf{H} \mathbf{R}+\mathbf{R} \mathbf{H} \mathbf{R}^{2}\right)\right.} \\
& +\left(I_{1} I_{2}-I_{3}\right)\left(\mathbf{R}^{2} \mathbf{H}+\mathbf{H R}^{2}\right) \\
& +\left(I_{1}^{3}+I_{3}\right) \mathbf{R H R}-I_{1}^{2} I_{2}(\mathbf{R H}+\mathbf{H R}) \\
& \left.+\left(I_{1}^{2} I_{3}+I_{2}\left(I_{1} I_{2}-I_{3}\right)\right) \mathbf{H}\right],
\end{aligned}
$$

where $I_{1}, I_{2}$, and $I_{3}$ are the principal invariants of $\mathbf{R}$,

$$
\begin{gathered}
I_{1}=R_{i i}, \quad I_{2}=\left(R_{i i} R_{j j}-R_{i j} R_{i j}\right) / 2, \\
I_{3}=\left(R_{i i} R_{j j} R_{k k}-3 R_{i i} R_{j k} R_{j k}+2 R_{i k} R_{j l} R_{k l}\right) / 6 .
\end{gathered}
$$

$B_{i}$ 's are then explicitly determined by Eq. (32), i.e.,

$$
B_{i}=\frac{1}{\tau_{v}}\left(\left\langle\widetilde{u_{i}}\right\rangle^{\dagger}-\left\langle\widetilde{u_{j}}\right\rangle\right)-A_{i j}\left\langle\widetilde{u_{j}}\right\rangle,
$$

such that the ALF term in Eq. (22) is completely defined.

It can be noticed that Eqs. (35) and (37) only depend on the statistical moments of filtered velocity $\widetilde{u_{i}}$, in accordance with the definition of $A_{i j}$ and $B_{i}$ as deterministic coefficients. Relaxation times $\tau_{r}$ and $\tau_{v}$ are adjustable parameters that control the intensity of the ALF.

\section{Restriction to the isotropic case and link with the linear forcing of Lundgren}

The specific case of homogeneous isotropic turbulence (HIT) is now considered. Specifically, an initially isotropic flow is considered and the target statistics of the forcing are defined as

$$
\begin{aligned}
\left\langle\tilde{u}_{i}\right\rangle^{\dagger} & =0, \\
\left\langle u_{i}^{\prime} u_{j}^{\prime}\right\rangle^{\dagger} & =\frac{2}{3} k_{r}^{\dagger} \delta_{i j},
\end{aligned}
$$

where $k_{r}=\frac{1}{2}\left\langle u_{i}^{\prime} u_{i}^{\prime}\right\rangle$ denotes the resolved turbulent energy. In that case, the flow necessarily remains statistically isotropic, such that the turbulent statistics are characterized by a single quantity, $k_{r}$,

$$
\begin{aligned}
\left\langle\widetilde{u}_{i}\right\rangle & =0, \\
\left\langle u_{i}^{\prime} u_{j}^{\prime}\right\rangle & =\frac{2}{3} k_{r} \delta_{i j} .
\end{aligned}
$$

Substituting Eqs. (39) and (41) into Eq. (33), it can be seen that the production of the forcing $P_{i j}^{f}$ is

$$
\frac{4}{3} k_{r} A_{i j}=\frac{1}{\tau_{r}}\left(\frac{2}{3} k_{r}^{\dagger}-\frac{2}{3} k_{r}\right) \delta_{i j}
$$

and the $A_{i j}$ coefficients are given by

$$
A_{i j}=\frac{1}{2 \tau_{r}}\left(\frac{k_{r}^{\dagger}}{k_{r}}-1\right) \delta_{i j} .
$$

Moreover, Eqs. (32), (38), and (40) imply that $\left\langle f_{i}\right\rangle=0$, such that

$$
f_{i}=\frac{1}{2 \tau_{r}}\left(\frac{k_{r}^{\dagger}}{k_{r}}-1\right) u_{i}^{\prime} .
$$

This isotropic restriction of the ALF, denoted as the Isotropic Linear Forcing (ILF) below, is a variant of the linear forcing term of the form

$$
f_{i}=A u_{i}^{\prime}
$$


proposed by Lundgren ${ }^{19}$ in order to generate a stationary isotropic turbulence. Since the turbulent energy equation, including the production due to this forcing, writes

$$
\frac{\partial k}{\partial t}=-\varepsilon+2 A k
$$

equilibrium is obtained for

$$
A=\frac{\varepsilon}{3 u_{\mathrm{rms}}^{\prime 2}} \text {. }
$$

The intensity of Lundgren's forcing is thus fixed in order to balance dissipation, such that turbulence remains at equilibrium, while the ILF is a restoring force that tends to bring turbulence back toward equilibrium.

Introducing a forcing term is standard in numerical studies of isotropic turbulence but, in the majority of cases, the forcing is defined in Fourier space and is restricted to low wavenumbers (see the review of Rosales and Meneveau ${ }^{20}$ ). In contrast, the linear forcing of Lundgren ${ }^{19}$ is applied in physical space and affects the whole wavenumber range, such that it can be easily applied to standard CFD codes.

Rosales and Meneveau ${ }^{20}$ extensively analyzed the properties of linearly forced isotropic turbulence (summarized in Sec. III A). The authors directly prescribed the $A$ coefficient in Eq. (45), inherently prescribing the integral time scale $k / \varepsilon$. Alternatively, they verified that a constant dissipation rate $\varepsilon^{\dagger}$ can be imposed in the numerator of Eq. (47) while the denominator $u_{\mathrm{rms}}^{\prime}$ is evaluated during the computation. Since, at equilibrium, $\varepsilon^{\dagger}$ corresponds to the rate of energy produced by the forcing, this approach is very similar to the one leading to the ILF (and, more generally, to the ALF). Since the ILF corresponds to replacing the coefficient $A$ of Eq. (47) by

$$
A=\frac{1}{2 \tau_{r}}\left(\frac{k_{r}^{\dagger}}{k_{r}}-1\right)
$$

the production of the forcing is not directly prescribed but adjusts to the $k_{r}^{\dagger}$ and $\tau_{r}$ parameters. At equilibrium, this production is balanced by dissipation such that

$$
\varepsilon=\frac{1}{\tau_{r}}\left(k_{r}^{\dagger}-k_{r}\right)
$$

Substituting Eq. (49) into Eq. (48), expression (47) of Lundgren's forcing is recovered.

\section{APPLICATION TO HOMOGENEOUS TURBULENCE}

\section{A. Homogeneous isotropic case}

In the present section, the ALF is validated in the case of HIT. The domain is a triply periodic box of size $L^{3}=(2 \pi)^{3}$, discretized by $N^{3}=32^{3}$ cells. The flow is initialized using an isotropic turbulent field generated by applying a spatial filter to an instantaneous DNS flow field ${ }^{27}$ at $R e_{\lambda}=104.5$. The subgrid stresses are modeled by the Smagorinsky model,

$$
\tau_{i j}^{d}=-2\left(C_{s} \widetilde{\Delta}\right)^{2}|\widetilde{S}| \widetilde{S}_{i j}, \quad|\widetilde{S}|=\sqrt{2 \widetilde{S}_{i j} \widetilde{S}_{i j}},
$$

where $\tau_{i j}^{d}=\tau_{i j}-\frac{1}{3} \tau_{k k} \delta_{i j}$ is the deviatoric part of the subgrid tensor, $C_{s}=0.18$ is the Smagorinsky constant, and $\widetilde{\Delta}=L / N$ is the filter width, equal to the grid step.

The computations presented throughout this paper are performed with the open source CFD software Code_Saturne developed by EDF (http://www.code-saturne.org). The solver is based on a finite volume method with a collocated arrangement on unstructured grids. Velocity-pressure coupling is performed using a predictor-corrector algorithm similar to the SIMPLEC method (see, for instance, Ref. 22). The time discretization is second order accurate, with a Crank-Nicholson scheme for the time derivative and a second order extrapolation of the source terms. The spatial discretization of the convective fluxes is centered and second order accurate. All linear systems are solved by iterative processes. In this paper, the Jacobi method is used for all variables except the 
pressure during the correction step, which is solved by a multi-grid procedure and the conjugate gradient method.

For the ILF method described in Sec. II D, the isotropy of the statistics is assumed a priori, such that it is only parametrized (see Eq. (44)) by the relaxation time scale $\tau_{r}$ and the target resolved turbulent energy $k_{r}^{\dagger}$. Here, the flow is forced to its initial resolved energy level, $k_{r}^{\dagger}=k_{0}$. In contrast, the ALF does not assume a priori the isotropy of the resolved statistics but brings them toward the isotropic target Reynolds stresses,

$$
\left\langle u_{i}^{\prime} u_{j}^{\prime}\right\rangle^{\dagger}=\frac{2}{3} k_{0} \delta_{i j}
$$

In both approaches, no explicit forcing of the mean flow is taken into account in this homogeneous case, which corresponds to the limit $\tau_{v} \rightarrow \infty$ in Eq. (32). The operator used to estimate $\left\langle\widetilde{u}_{i}\right\rangle$ and $\left\langle u_{i}^{\prime} u_{j}^{\prime}\right\rangle$ is a spatial averaging over the domain. All the quantities are made non-dimensional using the initial turbulent energy level $k_{0}$ and the size of the domain $L$.

Figure 1 shows the time evolution of the resolved kinetic energy for three values of the dimensionless time scale of the Reynolds stress forcing: $\tau_{r}^{*}=0.01,0.05,0.1$. It is observed that, after a transient decay, the forcing drives the turbulent energy towards the target value, and a permanent state is reached. The turbulent energy oscillates around its time-averaged value, denoted by $\left\langle k_{r}\right\rangle_{t}$. This behavior is consistent with the observations made by Rosales and Meneveau ${ }^{20}$ using DNS. As expected, the results obtained with the ALF and with its isotropic restriction, the ILF, are similar but not identical, since the initial field is not perfectly isotropic. With both formulations of the linear forcing, the turbulent energy level sustained by the forcing in the permanent state is always below the target $k_{0}$, since the forcing vanishes when the target is approached. The ratio $k_{r} / k_{0}$ monotonically depends on the relaxation time scale $\tau_{r}^{*}$.

The time evolution of the resolved energy spectrum $E(\kappa)$ is presented in Figs. 2 and 3. For the sake of visibility, the evolutions during the decay (before the energy reaches its minimum) and during the growth phase (until the permanent state is reached) are plotted in separated graphs in Fig. 2.

During the initial energy decay (Fig. 2(a)), the energy decreases rapidly within the high wavenumber range $(\kappa>4)$ whilst the largest eddies sustain their energy. Then the decrease at high wavenumbers slows down and energy at low wavenumbers starts to increase. In the growth phase (Fig. 2(b)), the forcing leads to a strong gain of energy in the low wavenumber range. In contrast, energy decreases in the high wavenumber range. It is worth noting that, by definition, the linear forcing acts on all the wavenumbers, as can be seen in the equation for the energy spectrum, ${ }^{19}$

$$
\left(\frac{\partial}{\partial t}+2 v \kappa^{2}-2 A\right) E(\kappa)=T(\kappa)
$$

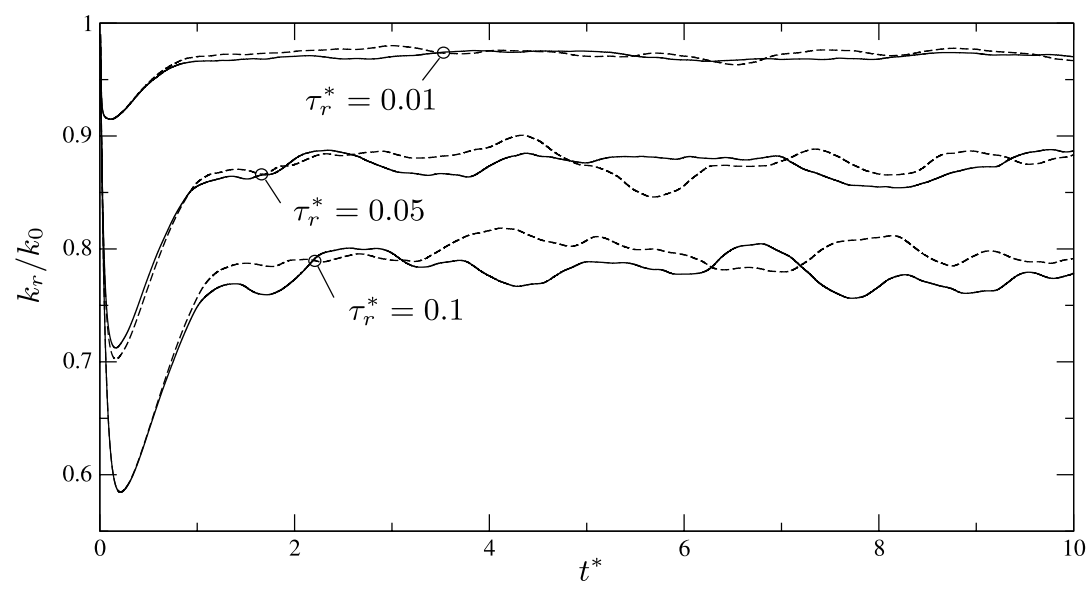

FIG. 1. Time evolution of turbulent kinetic energy. Forcing of a HIT toward its initial energy level $k_{0}$ for $\tau_{r}^{*}=0.01,0.05,0.1$. Black solid line: ALF; dashed lines: ILF. 

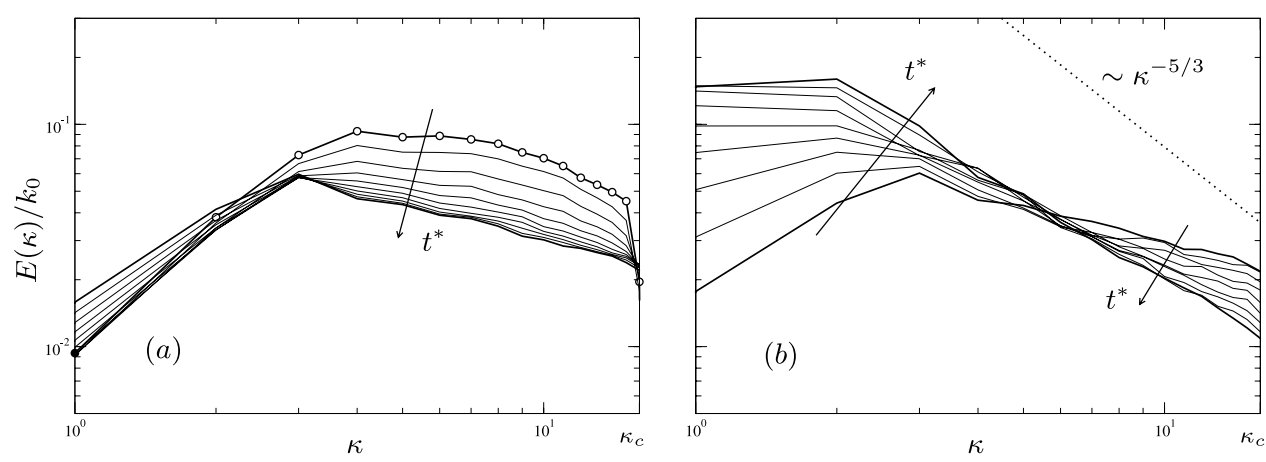

FIG. 2. Evolution of the kinetic energy spectrum during the transient phase. Forcing of a HIT towards its initial energy level $k_{0}$ for $\tau_{r}^{*}=0.1$. (a) Decreasing phase $\left(0 \leq t^{*} \leq 0.18\right.$, lines are plotted at intervals of $\left.\delta t^{*}=0.02\right)$, (b) increasing phase, $\left(0.2 \leq t^{*} \leq 1, \delta t^{*}=0.1\right)$. The initial spectrum is indicated by the circles.

Once turbulence has reached a permanent state, the energy spectrum is stationary (Fig. 3). Here again, the results produced by the ALF and the ILF are very similar. Comparing graphs 3(a) and 3(b), it also appears that the global shape of the spectrum is independent of the forcing parameter $\tau_{r}$. The mean spectrum behaves as $\kappa^{-\alpha}$ on a wide wavenumber range, up to the cut-off wavenumber $\kappa_{c}$. As for the DNS of Lundgren ${ }^{19}$ and Rosales and Meneveau, ${ }^{20} \alpha$ is close to $5 / 3$ in the present LES, indicating that the linear forcing is compatible with an inertial cascade.

At equilibrium, Eq. (49) can be used to evaluate the integral time scale $T$ and the length scale $L_{\|}$ defined by

$$
T=\frac{\left\langle u_{\mathrm{rms}}^{\prime 2}\right\rangle_{t}}{\varepsilon}, \quad L_{\|}=\frac{\left\langle u_{\mathrm{rms}}^{\prime 3}\right\rangle_{t}}{\varepsilon},
$$

where $u_{\mathrm{rms}}^{\prime}=\sqrt{2\left\langle k_{r}\right\rangle_{t} / 3}$ is the rms of the resolved velocity. The results are reported in Table I.

It is observed that the integral time scale $T$ slightly depends on the forcing parameter $\tau_{r}$. The dependency of $T$ on $\tau_{r}$ is not trivial, since $A$ in Eq. (48) is a function of $1 / \tau_{r}$ and is also implicitly dependent on $k_{0} / k_{r}$, as shown in Fig. 1. Table I indicates that the forcing parameter $\tau_{r}$ globally exhibits a moderate influence on the integral time scale $T$.

\section{B. Control of the integral length scale}

Rosales and Meneveau, ${ }^{20}$ showed, using dimensional analysis, that the linear forcing introduces a time scale into the system but no length scale. In essence, this leaves only the box size $L$ as an available characteristic scale for the large scales to fix the dissipation rate: $\varepsilon \sim A^{3} L^{2}$. Indeed, it can
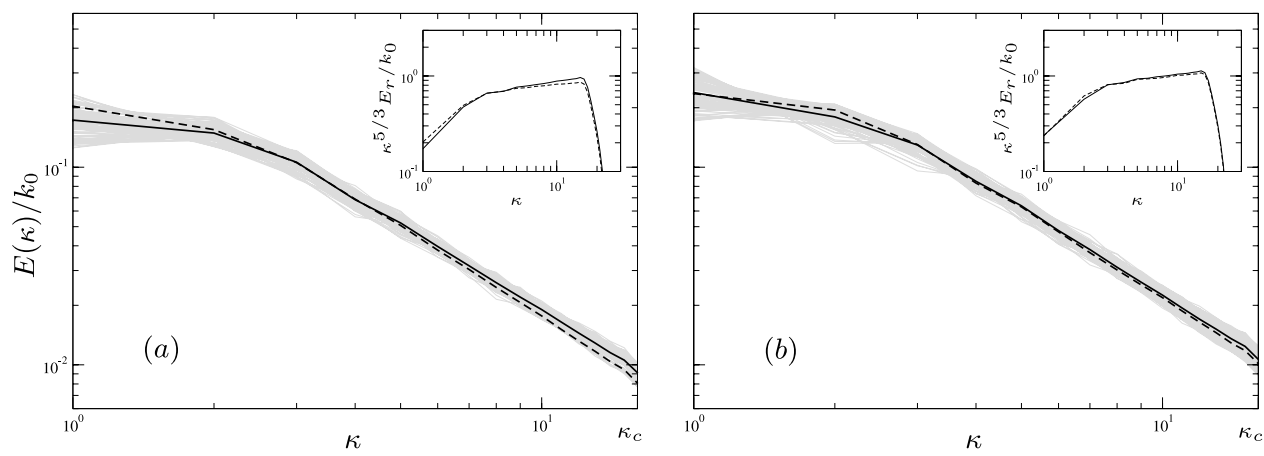

FIG. 3. Evolution of the kinetic energy spectrum in the permanent state. Forcing of a HIT towards its initial energy level $k_{0}$ for (a) $\tau_{r}^{*}=0.1$, (b) $\tau_{r}^{*}=0.01$. Grey lines: 100 spectra uniformly distributed in the $3<t^{*} \leq 10$ time interval. Black lines: time-averaged spectrum (multiplied by $\kappa^{5 / 3}$ in the inset); black solid line: ALF; dashed lines: ILF. 
TABLE I. Mean resolved kinetic energy, integral time scale $T$, and length scale $L_{\|}$in Eq. (53) (time averaging over the time interval $\left.3<t^{*} \leq 40\right)$.

\begin{tabular}{|c|c|c|c|c|c|c|}
\hline \multirow[b]{2}{*}{$\tau_{r}^{*}$} & \multicolumn{2}{|c|}{$\left\langle k_{r}\right\rangle / k_{0}$} & \multicolumn{2}{|c|}{$T^{*}$} & \multicolumn{2}{|c|}{$L_{\|}^{*}$} \\
\hline & ALF & ILF & ALF & ILF & ALF & ILF \\
\hline 0.01 & 0.970 & 0.972 & 0.217 & 0.230 & 0.174 & 0.185 \\
\hline 0.05 & 0.874 & 0.883 & 0.232 & 0.253 & 0.177 & 0.194 \\
\hline 0.1 & 0.787 & 0.797 & 0.246 & 0.262 & 0.178 & 0.191 \\
\hline
\end{tabular}

be observed in Fig. 2 that the integral length scale of the initial field is not preserved by the forcing method but grows up to the box size, as already observed by Rosales and Meneveau. ${ }^{20}$ Using Eq. (47), this leads to

$$
\frac{u_{\mathrm{rms}}^{\prime 3}}{\varepsilon} \sim L
$$

Table I shows that $L_{\|}$is virtually unaffected by the particular choice of $\tau_{r}$, thus confirming that the forcing method does not influence the integral length scale. The proportionality constant $L_{\|} / L$ reported by Rosales and Meneveau ${ }^{20}$ is 0.19 , in close agreement with the present LES results.

For cases more general than homogeneous turbulence, it is desirable to prescribe the length scale of the synthetic turbulence, independently of the size of the domain. This can be done using a slight modification of the forcing scheme, which aims at forcing only the scales smaller than a length scale $L_{f}$. The forcing is therefore rewritten as

$$
f_{i}=A_{i j}\left(\widetilde{u}_{j}-\widehat{\widetilde{u}}_{j}\right)+B_{i},
$$

where $\widehat{\widetilde{u}}_{j}$ is simply determined by explicitly filtering the resolved velocity $\widetilde{u}_{i}$. A second order differential elliptic filter is chosen (e.g., Germano, ${ }^{28}$ Bose et al.,${ }^{29}$ ) because of its easy implementation in any solver,

$$
\widehat{\widetilde{u}}_{i}-\nabla \cdot\left(L_{f}^{2} \nabla \widehat{\widetilde{u}}_{i}\right)=\widetilde{u}_{i},
$$

where $L_{f}$ is the filter width.

Consequently, the mean part of the force, which contributes to the mean motion, reads

$$
\left\langle f_{i}\right\rangle=A_{i j}\left(\left\langle\widetilde{u}_{j}\right\rangle-\left\langle\widehat{\widetilde{u}}_{j}\right\rangle\right)+B_{i},
$$

and the production term that arises in the resolved stress equation is

$$
P_{i j}^{f}=A_{i k}\left(\left\langle u_{k}^{\prime} u_{j}^{\prime}\right\rangle-\left\langle u_{k}^{\prime \prime} u_{j}^{\prime}\right\rangle\right)+A_{j k}\left(\left\langle u_{k}^{\prime} u_{i}^{\prime}\right\rangle-\left\langle u_{k}^{\prime \prime} u_{i}^{\prime}\right\rangle\right),
$$

where $\left\langle u_{k}^{\prime \prime} u_{j}^{\prime}\right\rangle$ is the cross-correlation between the fluctuating part of the resolved velocity $u_{i}^{\prime}={\widetilde{u_{i}}}_{-}$ $\left\langle\widetilde{u_{i}}\right\rangle$ and the fluctuating part of the filtered resolved velocity $u_{i}^{\prime \prime}=\widehat{\widetilde{u_{i}}}-\left\langle\widehat{\widetilde{u}_{i}}\right\rangle$.

As described in Sec. II C, the coefficients $A_{i j}$ are computed at each point using constraint (31), and constraint (30) provides the coefficients $B_{i}$. The force $f_{i}$ then becomes

$$
f_{i}=A_{i j}\left(\left(\widetilde{u}_{j}-\widehat{\widetilde{u}}_{j}\right)-\left(\left\langle\widetilde{u}_{j}\right\rangle-\left\langle\widetilde{\widetilde{u}}_{j}\right\rangle\right)\right)+\frac{1}{\tau_{v}}\left(\left\langle\widetilde{u}_{i}\right\rangle^{\dagger}-\left\langle\widetilde{u}_{i}\right\rangle\right) .
$$

To characterize the integral length scale $L_{\|}$as a function of $L_{f}$, two series of tests have been carried out, using two different boxes of widths $L=2 \pi$ and $L=4 \pi$, with the same grid step $\Delta x=2 \pi / 64$. For each grid, eight simulations are carried out with different $L_{f}$ varying between $L_{f}=0.6$ and $L_{f}=2.5$. For all simulations, the forcing time scales are $\tau_{v}^{*}=\tau_{r}^{*}=0.1$ and the target resolved stresses are the same as in Sec. III A.

Long-time averaged spectra are shown in Fig. 4. It is clearly seen that the spectra are shifted toward the high wavenumbers when $L_{f}$ is decreased. It is also noticeable that the level of the spectra, and hence the resolved kinetic energy $k_{r}$ also decreases with $L_{f}$. The main conclusion that can be 


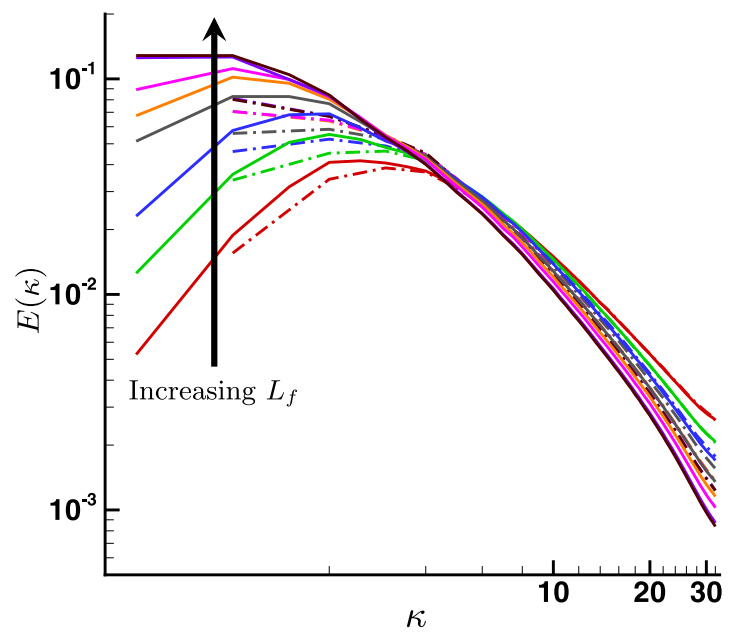

FIG. 4. Averaged spectra of the velocity field achieved using different values of $L_{f}(0.6 ; 0.8 ; 1 ; 1.3 ; 1.6 ; 1.9 ; 2.2 ; 2.5)$. Solid lines: $L=4 \pi$; dashed lines: $L=2 \pi$.

drawn from this figure is that, when $L_{f}$ is sufficiently small compared to the size of the box $L$, the spectra obtained in the two boxes, $L=2 \pi$ and $L=4 \pi$, are very similar, which shows that the modified forcing is able to impose the integral length scale of the generated turbulence via the filtering length scale $L_{f}$, independently of the size of the box $L$.

It is also observed that, for large $L_{f}$, the sequence of spectra obtained in the small box approaches a limit spectrum, because the size of the box imposes an upper constraint to the integral length scale $L_{\|}$. This length scale, computed from Eq. (53), using Eq. (49) to evaluate the dissipation, is plotted in Figure 5(a). In the small box, it is confirmed that $L_{\|}$remains bounded for large $L_{f}$. Since the effect of the filter vanishes when $L_{f}$ goes to infinity, $L_{\|}$is asymptotic to the horizontal line $0.19 L$ that corresponds to the value obtained without filtering in Sec. III A.

In the case of the large box $(L=4 \pi)$, the integral scale is approximately proportional to $L_{f}$,

$$
L_{\|} \simeq 0.7 L_{f} .
$$

Using this relation, Eq. (49) becomes

$$
\frac{1}{\tau_{r}}\left(k_{r}^{\dagger}-k_{r}\right)=\varepsilon=\frac{u_{\mathrm{rms}}^{\prime 3}}{L_{\|}}=\frac{k_{r}^{\frac{3}{2}}}{\left(\frac{3}{2}\right)^{\frac{3}{2}} L_{\|}} \simeq \frac{k_{r}^{\frac{3}{2}}}{1.3 L_{f}},
$$
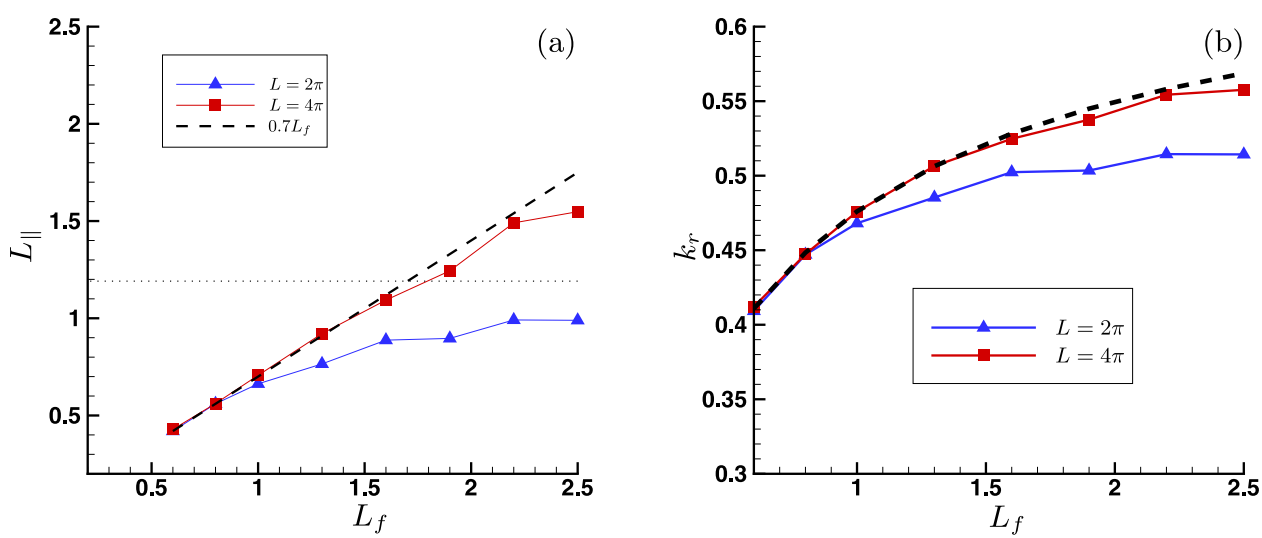

FIG. 5. (a) Integral length scale $L_{\|}$as a function of the filtering length scale $L_{f}$ for two different sizes $L$ of the box. The dashed line represents the equation $L_{\|}=0.7 L_{f}$. The dotted line is the asymptote $0.19 L$ for $L=2 \pi$ achieved when $L_{f} \rightarrow \infty$ (no filtering). (b) Turbulent kinetic energy as a function of $L_{f}$ for two different sizes $L$ of the box. The dashed line represents the solution of Eq. (61). 
such that $k_{r}$ can be computed from the equation

$$
k_{r}\left(1+\frac{\tau_{r}}{1.3 L_{f}} k_{r}^{\frac{1}{2}}\right)=k_{r}^{\dagger}
$$

It is shown in Figure 5(b) that the solution of this equation is in good agreement with the values obtained in the large box, as long as $L_{f}$ remains sufficiently small compared to $L$.

The modified forcing methods (55) and (56) thus make the control of the integral length scale possible. This method is applied in the case of a turbulent channel flow in Sec. IV.

\section{Homogeneous anisotropic case}

The flexibility and effectiveness of the ALF is now demonstrated in anisotropic cases. The configuration is the same as in Sec. III A, except for the target stresses that are now anisotropic. Introducing the anisotropy tensor

$$
b_{i j}=\frac{\left\langle u_{i}^{\prime} u_{j}^{\prime}\right\rangle}{2 k}-\frac{1}{3} \delta_{i j}
$$

and its second and third invariants

$$
\text { II }=-\frac{1}{2} b_{i j} b_{i j}, \quad \text { III }=\frac{1}{3} b_{i j} b_{j k} b_{k i},
$$

the anisotropies considered in this section are plotted on the invariant $(I I I,-I I)$ map (the so-called Lumley triangle $\mathrm{e}^{30,31}$ ) in Fig. 6 . For the present test, the arbitrary target stresses successively imposed to the ALF are the following:

(i) a three-component anisotropic turbulence (the point denoted by "O" in Fig. 6)

$$
\begin{gathered}
\left\langle u^{\prime 2}\right\rangle^{\dagger}=\frac{1}{3} k_{0}, \quad\left\langle v^{\prime 2}\right\rangle^{\dagger}=\frac{2}{3} k_{0}, \quad\left\langle w^{\prime 2}\right\rangle^{\dagger}=k_{0}, \\
\left\langle u^{\prime} v^{\prime}\right\rangle^{\dagger}=\left\langle u^{\prime} w^{\prime}\right\rangle^{\dagger}=\left\langle v^{\prime} w^{\prime}\right\rangle^{\dagger}=0,
\end{gathered}
$$

(ii) a nearly two-component axisymmetric turbulence (close to the " $2 \mathrm{C}$-axi" corner of the triangle)

$$
\begin{gathered}
\left\langle u^{2}\right\rangle^{\dagger}=\left\langle v^{\prime 2}\right\rangle^{\dagger}=0.99 k_{0}, \quad\left\langle w^{2}\right\rangle=0.02 k_{0}, \\
\left\langle u^{\prime} v^{\prime}\right\rangle^{\dagger}=\left\langle u^{\prime} w^{\prime}\right\rangle^{\dagger}=\left\langle v^{\prime} w^{\prime}\right\rangle^{\dagger}=0,
\end{gathered}
$$

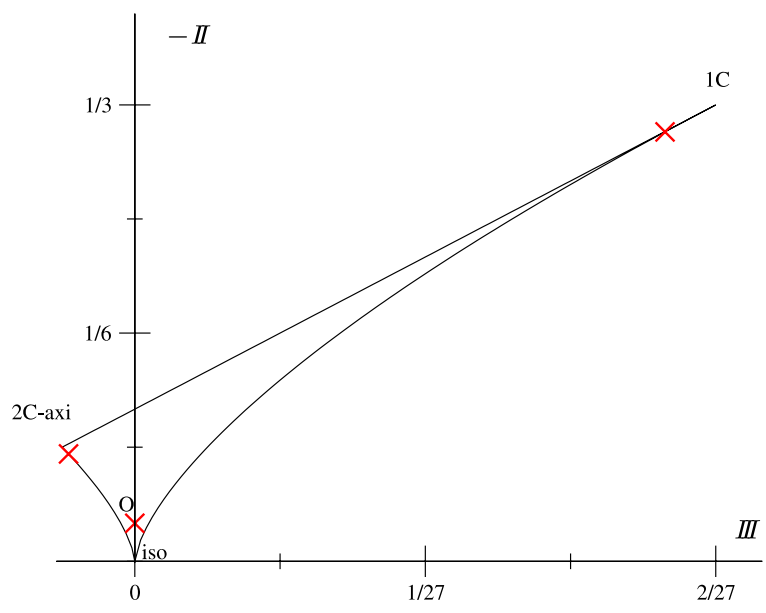

FIG. 6. Lumley triangle in the (III, - II ) invariant plane. The red crosses indicate the target anisotropies imposed to the ALF, Eqs. (65)-(67). 
(iii) a nearly one-component axisymmetric turbulence (close to the "1C" corner of the triangle)

$$
\begin{gathered}
\left\langle u^{\prime 2}\right\rangle^{\dagger}=1.96 k_{0}, \quad\left\langle v^{\prime 2}\right\rangle^{\dagger}=\left\langle w^{\prime 2}\right\rangle^{\dagger}=0.02 k_{0}, \\
\left\langle u^{\prime} v^{\prime}\right\rangle^{\dagger}=\left\langle u^{\prime} w^{\prime}\right\rangle^{\dagger}=\left\langle v^{\prime} w^{\prime}\right\rangle^{\dagger}=0 .
\end{gathered}
$$

It is worth noting that, as demonstrated in Appendix A, the ALF is frame invariant. Therefore, it is sufficient to show that the considered anisotropies can be imposed in the coordinate frame aligned with the principal directions of Reynolds tensor, as in Eqs. (65)-(67).

Considering the three-component state of Eq. (65), Fig. 7(a) shows that after a short decreasing phase, the resolved turbulent kinetic energy $k_{r}$ fluctuates around a constant value which is closer to the target energy $k_{0}$ when the forcing parameter $\tau_{r}$ is small. As regards the anisotropy, Fig. 7(b) indicates, in particular for $\tau^{*}=0.05$, that the gaps between the observed and the target Reynolds stresses are not the same for the different components. Actually, it can be seen in Fig. 7(c) that the absolute value of the components of the anisotropy is slightly underestimated,

$$
\left|\left\langle b_{i j}\right\rangle_{t}\right|=\left(1-\epsilon\left(\tau_{r}\right)\right)\left|b_{i j}^{\dagger}\right|,
$$

where $\epsilon$ is an error function. This relationship implies that the long-time averaged second invariant $\langle-I I\rangle_{t}$ is lower than its target value $-I^{\dagger}$, while the long-time averaged third invariant $\langle I I I\rangle_{t}$ is zero, as shown in Fig. 7(d).

Figures 8 and 9 present the results obtained in the nearly two-component (Eq. (66)) and onecomponent (Eq. (67)) cases, respectively. Figures 8(a) and 9(a) show that, similar to the previous case, the Reynolds stresses fluctuate around constant values, and Figs. 8(b) and 9(b) confirm that the target anisotropy is asymptotically reached. In comparison with previous cases, the transient phase, before the permanent state is reached, is longer, and in the permanent state, the amplitude of the fluctuations around the equilibrium values is smaller. Figs. 8(c) and 9(c) illustrate the typical flow structures obtained in these cases of nearly two-component and one-component axisymmetric forcings.

A last case is now considered to demonstrate the effectiveness of the ALF: starting from an isotropic state, the nearly two-component and one-component axisymmetric anisotropic states (Eqs. (66) and (67)) are successively enforced and, eventually, the flow is forced back to isotropy (this cyclic case can be seen as a tour of the Lumley triangle). To that end, the target stresses of the ALF are abruptly switched during the computation. It is visible in Fig. 10(a) that the flow rapidly adjusts to the desired anisotropic state. Moreover, it can be seen in the (III,-II) map (Fig. 10(b)) that the turbulent anisotropy passes along the boundaries of the Lumley triangle, without introducing artificial three-component states during the transient. This case also shows the robustness of the ALF to quickly adapt to target statistics.

\section{APPLICATION TO TURBULENT CHANNEL FLOWS}

Section III has demonstrated that the ALF method can successfully impose target statistics in the case of homogeneous turbulence. The purpose of the present section is to extend the method to spatially developing flows, for which the turbulent channel flow is prototypical. Before proceeding to the application of the method to hybrid RANS/LES computations, a parametric study is carried out in LES, using the Smagorinsky model, in order to identify the influence of the different parameters on the flow development toward the target solution.

\section{A. Parametric study}

The case of spatially developing turbulent channel flow at $R_{b}=U_{b} h / v=7000$, is now considered, where $h$ is the half-width of the channel and $U_{b}$ is the bulk velocity. The target statistics of the ALF are taken from a precursor periodic simulation with the same grid spacing. The imposed inlet velocity is the target mean velocity $\left\langle\widetilde{u_{i}}\right\rangle^{\dagger}$, without superimposed fluctuations. The whole flow domain is forced with the ALF, in order to investigate the spatial development of the flow. 


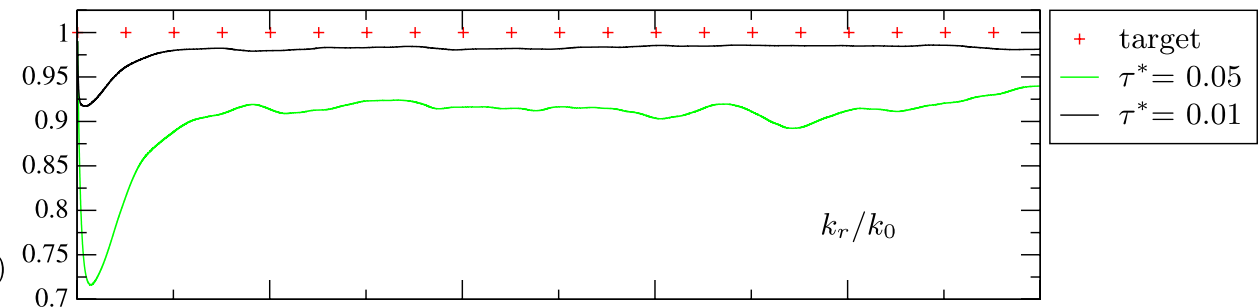

(b)

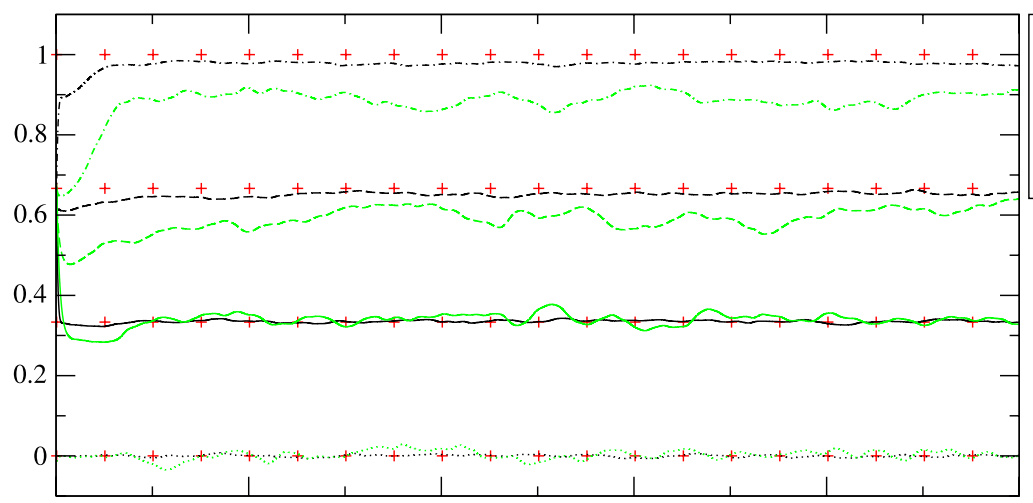

$$
\begin{array}{ll}
- & \left\langle u^{\prime 2}\right\rangle_{r} / k_{0} \\
--- & \left\langle v^{\prime 2}\right\rangle_{r} / k_{0} \\
\cdots & \left\langle w^{\prime 2}\right\rangle_{r} / k_{0} \\
\cdots \cdots & \left\langle u^{\prime} v^{\prime}\right\rangle_{r} / k_{0}
\end{array}
$$

(c)

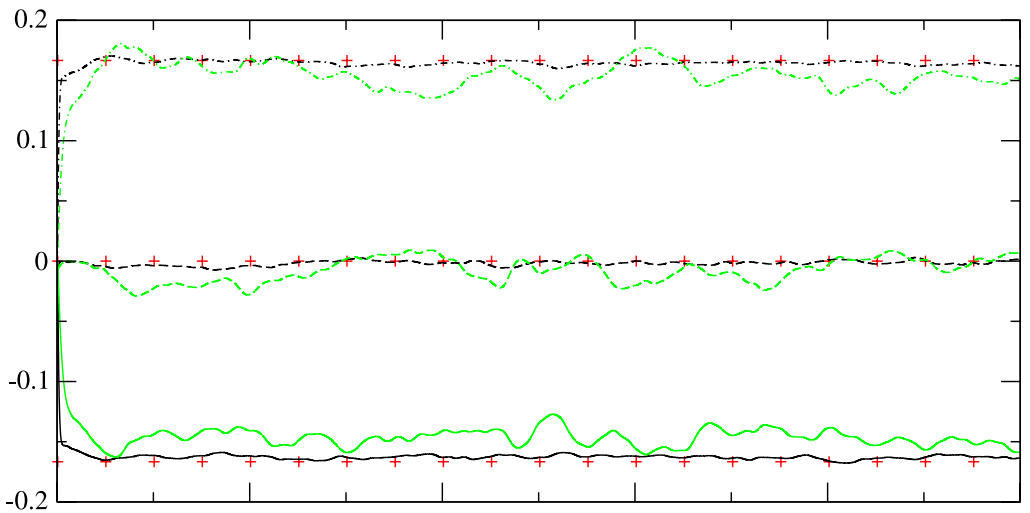

$$
\begin{array}{ll}
- & b_{11} \\
--- & b_{22} \\
\cdots & b_{33}
\end{array}
$$

$(d)$

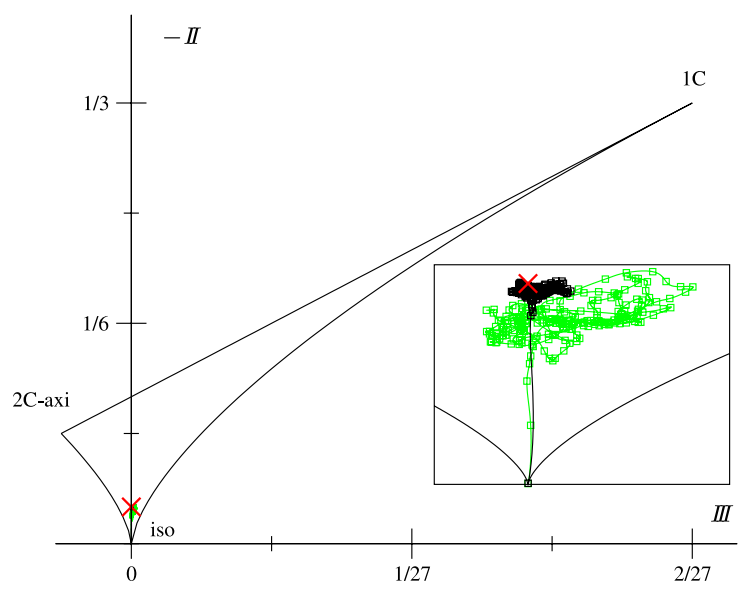

FIG. 7. Forcing of an initially isotropic turbulence toward the three-component anisotropic state of Eq. (65). Time evolution (a) of the turbulent kinetic energy, (b) of the Reynolds stress tensor, (c) of the anisotropy tensor, and (d) trajectory of the anisotropy in the $(I I I,-I I)$ invariant map (the inset is an enlargement in which the lines are marked every $\left.\delta t^{*}=0.05\right)$. Red + , red $\times$ : target values; green solid line: forced LES, $\tau_{r}^{*}=0.05$; black solid line: forced LES, $\tau_{r}^{*}=0.01$. 
(a)

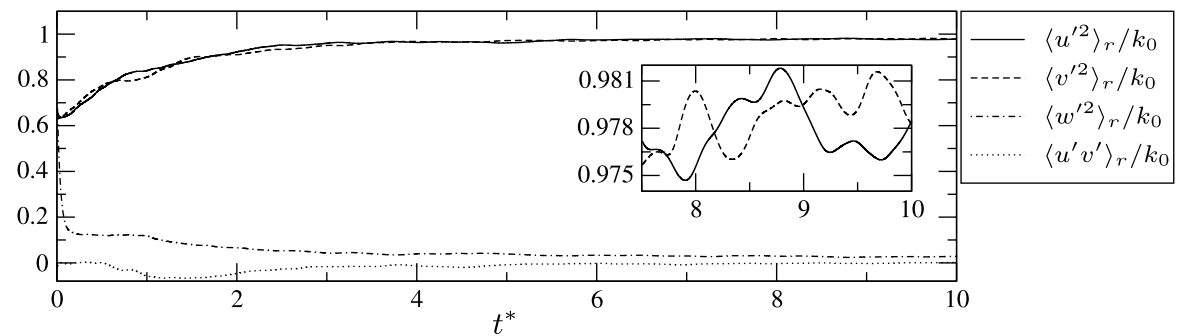

(b)

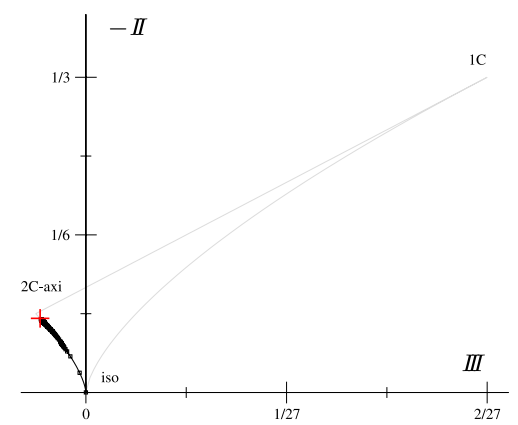

(c)

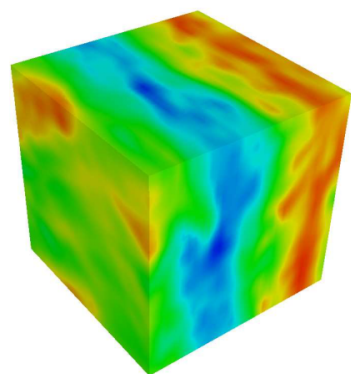

FIG. 8. Forcing of an initially isotropic turbulence toward the nearly two-component state of Eq. (66), $\tau_{r}^{*}=0.05$. (a) Evolution of the Reynolds stresses (the inset is a zoom); (b) trajectory of the anisotropy in the (III, - II ) invariant map (lines are marked every $\delta t^{*}=0.05$ time lapses; red +: target anisotropy); (c) visualization of the velocity $u^{\prime} / \sqrt{k_{0}}$ at $t^{*}=10$.

For the reference periodic simulation, the domain is limited to $3 \pi h \times 2 h \times \pi h$ and discretized with a $73 \times 86 \times 81$ cells, which corresponds to cells of size $x^{+}=50, z^{+}=15, y_{\min }^{+}=2.1$, and $y_{\max }^{+}=$ 12 in wall units.

For the spatially developing cases, a number of computations are carried out, in order to investigate the influence of the parameters involved in the ALF. The characteristics of the computations are summarized in Table II. Beside the relaxation time scales $\tau_{v}$ and $\tau_{r}$, the influence of imposing a length scale $L_{f}$ via a differential filter and of the choice of the approximate averaging operator is also discussed.

(a)

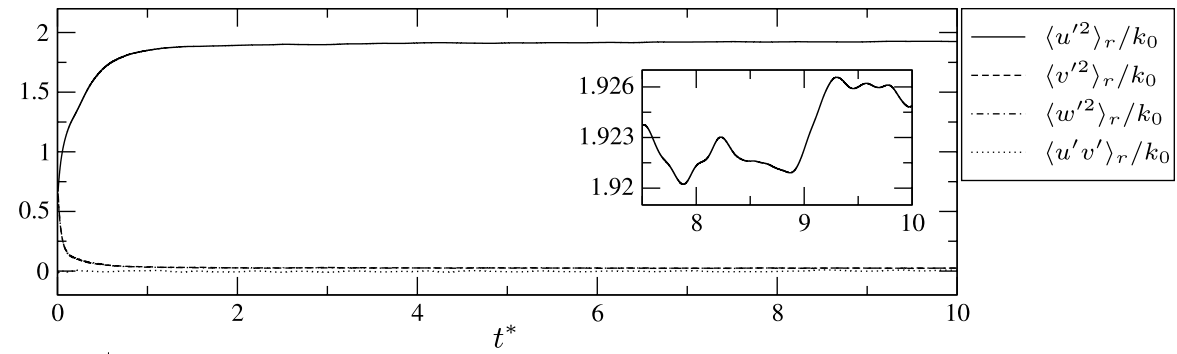

(b)

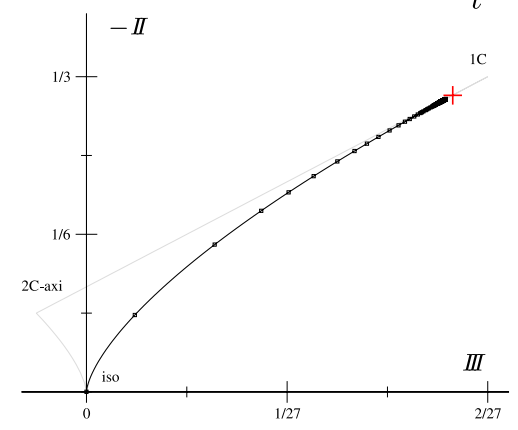

(c)

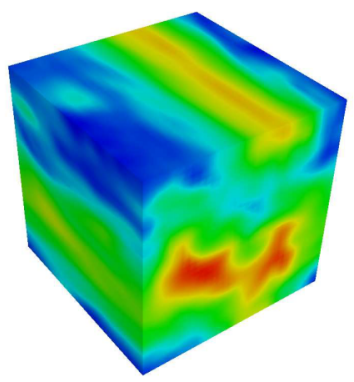

FIG. 9. Forcing of an initially isotropic turbulence toward the nearly one-component state of Eq. (67), $\tau_{r}^{*}=0.05$. Legend: see Fig. 8. 

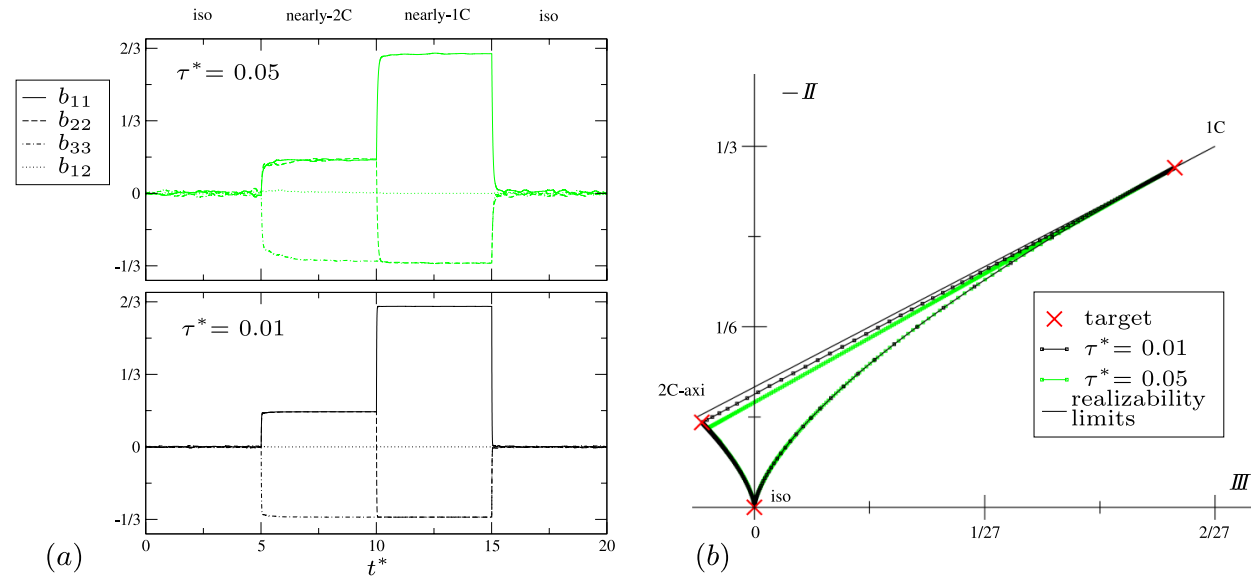

(b)

FIG. 10. (a) Time evolution of the anisotropy and (b) trajectory in the (III, - II ) invariant map.

For these computations, the size of the domain is $10 \pi h \times 2 h \times \pi h$ and spatial discretization uses the same grid spacing as for the reference periodic simulation. The forcing is applied starting from the first time step. The resolved flow statistics, involved in the ALF formulation, are evaluated using exponential time filter (9). For the sake of generality, the less favorable situation is considered, i.e., a flow without spatial homogeneous direction. Therefore, most of the computations do not exploit the homogeneity of the flow in the spanwise direction, except for specific tests. Since differential filtering requires initial conditions for the filtered moments, the target values are used. In contrast to the approximate moments used in the ALF, the statistics presented in the figure below are obtained by time averaging from $t=200 \mathrm{~h} / \mathrm{U}$ to $600 \mathrm{~h} / \mathrm{Ub}$ and spatial averaging in the homogeneous $z$ direction.

\section{Influence of an imposed length scale $L_{f}$}

In such a confined flow, it is expected that imposing a length scale $L_{f}$ through a differential filter, as presented in Sec. III B, is unnecessary, since the length scale is naturally imposed by the distance to the wall. In order to investigate the validity of this assumption, tests are carried out to compare computations using the ALF with and without the differential filter (cases 1a-d), using the parameters $\tau_{v}=\tau_{r}=0.5 h / U_{b}$.

For the forcing with filtering, the relevant width of the filter is evaluated from the reference periodic simulation: the turbulent kinetic energy budget is computed to estimate the dissipation rate $\varepsilon$ and a reference length scale is evaluated as

$$
L_{\mathrm{ref}}(y)=\frac{k_{r}^{\frac{3}{2}}}{\varepsilon} .
$$

Since computations of homogeneous turbulence in Sec. III B have shown that the integral length scale satisfies the relation $L_{\|}=0.7 L_{f}$, or equivalently $k_{r}^{3 / 2} / \varepsilon=1.3 L_{f}$, the filter width is chosen as $L_{f}(y)=C L_{\mathrm{ref}}(y) / 1.3$. Different values of the coefficient $C$ are applied in order to investigate the

TABLE II. Characteristics of computations using the ALF.

\begin{tabular}{llllr}
\hline \hline Case & \multicolumn{1}{c}{$\tau_{v}$} & \multicolumn{1}{c}{$\tau_{r}$} & \multicolumn{1}{c}{ Approximate averaging $\langle\cdot\rangle$} & Imposed $L_{f}$ \\
\hline $1 \mathrm{a}-\mathrm{d}$ & $0.5 h / U_{b}$ & $0.5 h / U_{b}$ & Gaussian filtering, $T=100+\langle\cdot\rangle_{z}$ & Yes \\
2 & $0.5 h / U_{b}$ & $0.5 h / U_{b}$ & Gaussian filtering, $T=100$ & No \\
3 & $0.1 h / U_{b}$ & $0.1 h / U_{b}$ & Gaussian filtering, $T=100$ & No \\
4 & $5 h / U_{b}$ & $0.1 h / U_{b}$ & Gaussian filtering, $T=100$ & No \\
5 & $5 h / U_{b}$ & $0.01 k / \varepsilon$ & Gaussian filtering, $T=100$ & No \\
$6 \mathrm{a}$ & $5 h / U_{b}$ & $0.01 k / \varepsilon$ & Exponential filtering, $T=10$ & No \\
$6 \mathrm{~b}$ & $5 h / U_{b}$ & $0.01 k / \varepsilon$ & Exponential filtering, $T=100+\langle\cdot\rangle_{z}$ & No \\
\hline \hline
\end{tabular}


influence of the length scale $L_{f}$ on the computed flow: $C=\infty$ (no filtering, case 1a), $C=1$ (case 1b), $C=0.5$ (case 1c), and $C=2$ (case 1d). It is worth mentioning that, in order to avoid numerical difficulties, the forcing term must be damped when $L_{f}$ is smaller than the cell size, which is in the case in a small region close to the wall.

For the sake of brevity, only profiles of the shear stress and turbulent kinetic energy at $x / h=5$ are shown in Figure 11. It is seen that all the profiles are virtually superimposed. The close examination of the statistical properties of the flow, such as spectra and two-point correlations (not shown here), has not revealed significant differences between the results of the different tests. Consequently, all the simulations presented in the rest of the article are carried out without filtering. It is worth mentioning that the relatively inaccurate prediction of the shear stress profile is due to the use of a large relaxation time $\tau_{r}$, as shown in Sec. IV A 2.

\section{Influence of the relaxation time scales $\tau_{v}$ and $\tau_{r}$}

In cases 2 and 3 (see Table II), the relaxation parameters for the mean velocity $\tau_{v}$ and the resolved Reynolds stresses $\tau_{r}$ are equal. Figure 12 presents the mean velocity, the turbulent kinetic energy, and the shear stress profiles at three locations downstream of the inlet: $x / h=2,5,10$. It is seen that, with the selected $\tau_{v}$ and $\tau_{r}$ values, the ALF rapidly generates turbulent stresses downstream of the inlet. It can be seen that the spatial evolution of the statistics is satisfactory: for the lower value of the relaxation parameters $\tau_{v}=\tau_{r}=0.1 h / U_{b}$, a short distance of $2 h$ is sufficient for the statistics to almost exactly adjust to their value corresponding to the fully developed state. For $\tau_{v}=\tau_{r}=0.5 h / U_{b}$, the development of the turbulent stresses is slower but, at $x / h=10$, the flow reaches the target statistics. Since the ALF is fully anisotropic, it is able to impose the anisotropy of the normal stresses $\left\langle u_{i}^{\prime 2}\right\rangle^{\dagger}$, as shown in Fig. 13.

In Fig. 14, the turbulent structures of the forced LES are shown in horizontal planes, at different distances from the wall. The turbulent structures of the resolved flow field are compared with those in the periodic reference case, the statistics of which are used as target moments. Coherent structures are rapidly generated downstream of the inlet with a spatial development of the turbulent structures more satisfactory for $\tau_{v}=\tau_{r}=0.1 \mathrm{~h} / U_{b}$ than for $\tau_{v}=\tau_{r}=0.5 \mathrm{~h} / U_{b}$. In particular, elongated near-wall structures do not appear directly downstream of the inlet for the latter case. Further from the walls and close to the inlet, it is observed that the structures generated are smaller than those of the fully developed flow. This phenomenon is limited, albeit still visible, for $\tau_{v}=\tau_{r}=0.1 h / U_{b}$. Furthermore, this phenomenon is observed on a short distance and the turbulent structures of the forced LES rapidly evolve toward an aspect very similar to that of the periodic LES.

The spectral content of the forced LES is shown in Fig. 15. Temporal Fourier transforms are obtained from a FFT algorithm, using samples of size $N=2^{13}$ during a time period of $409.6 h / U_{b}$. The temporal spectra of the velocity of the forced LES show a good agreement with those of the periodic simulation without forcing. An influence of $\tau_{v}$ on the low frequency fluctuations can be observed,
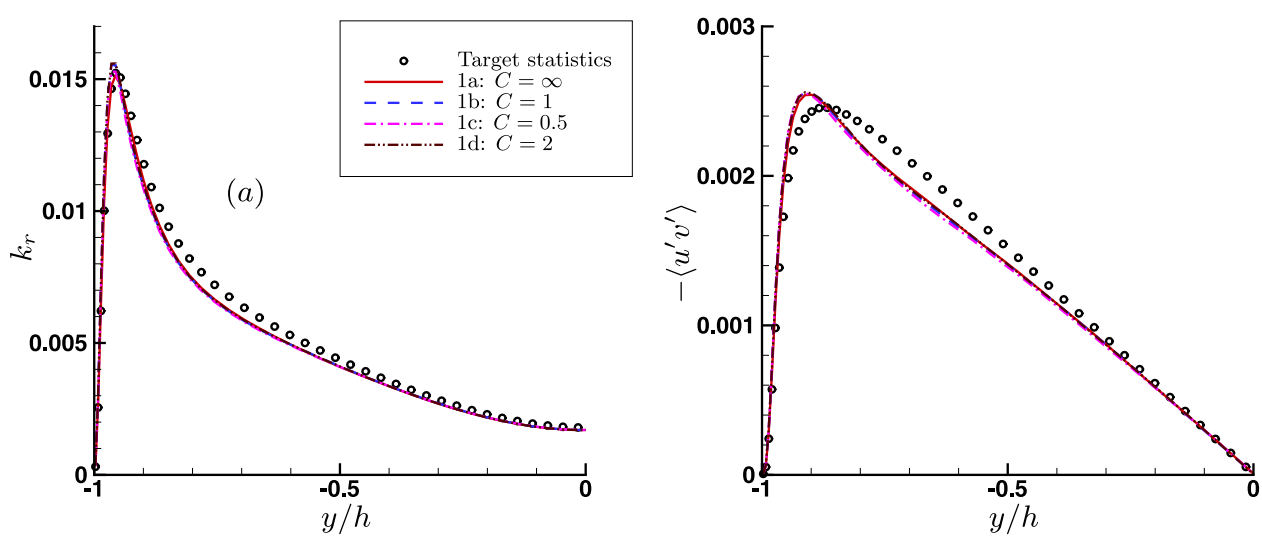

FIG. 11. (a) Resolved turbulent kinetic energy and (b) shear stress at $x / h=5$ obtained using different spatial filtering. $\tau_{v}=\tau_{r}=0.5 h / U_{b}$. 

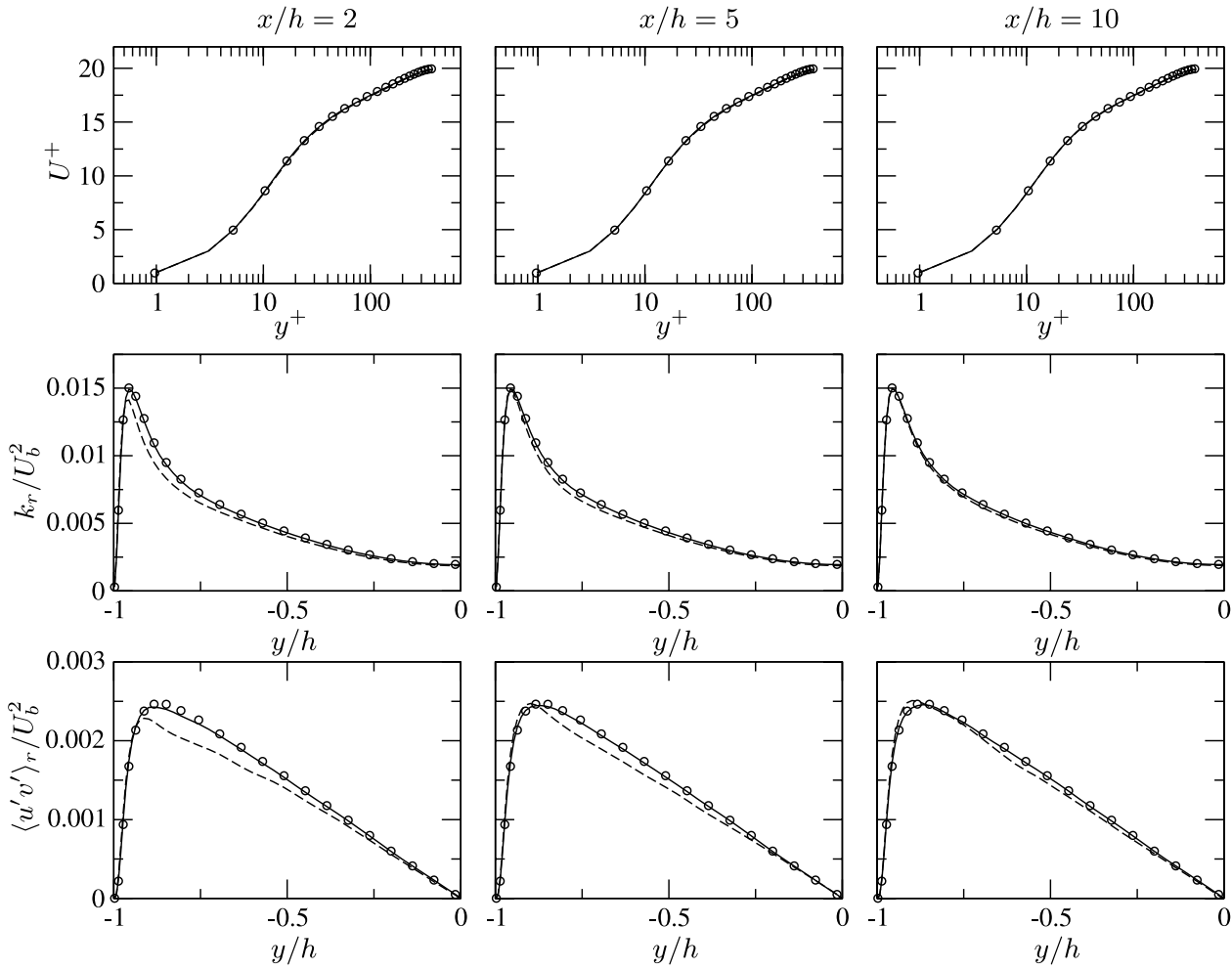

FIG. 12. Mean velocity, resolved turbulent kinetic energy, and turbulent shear stress at $x / h=2,5,10 . \circ$ : target statistics; dashed lines: forced LES, $\tau_{v}=\tau_{r}=0.5 h / U_{b}$ (case 2); black solid line: forced LES, $\tau_{v}=\tau_{r}=0.1 h / U_{b}$ (case 3).
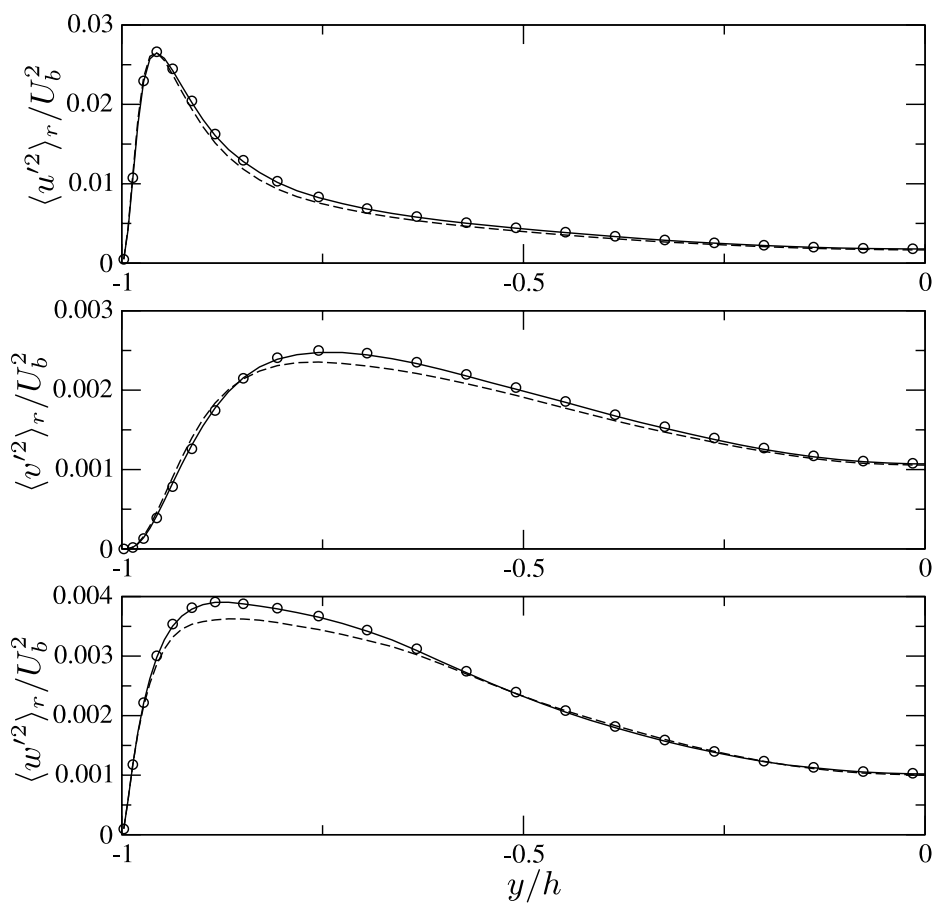

FIG. 13. Normal turbulent stresses at $x / h=5$. ०: target statistics; dashed lines: forced LES, $\tau_{v}=\tau_{r}=0.5 h / U_{b}($ case 2); black solid line: $\tau_{v}=\tau_{r}=0.1 \mathrm{~h} / U_{b}$ (case 3 ). 
(a)

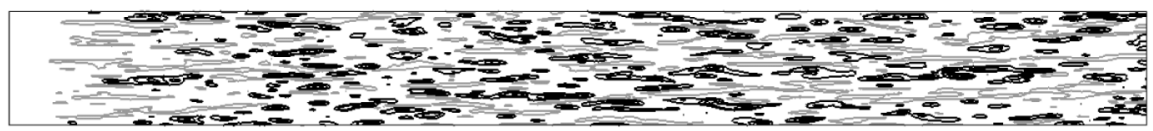

(b)

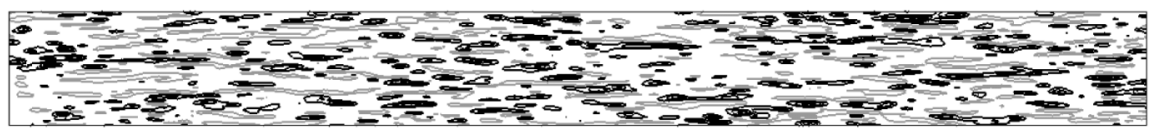

(c)

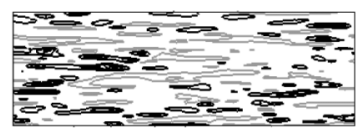

(d)

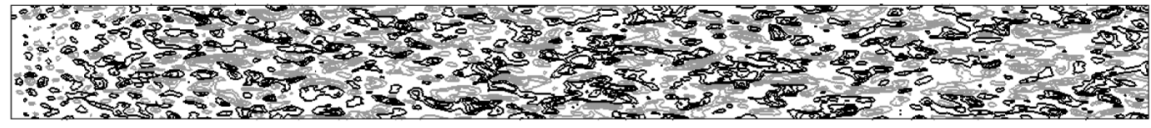

(e)

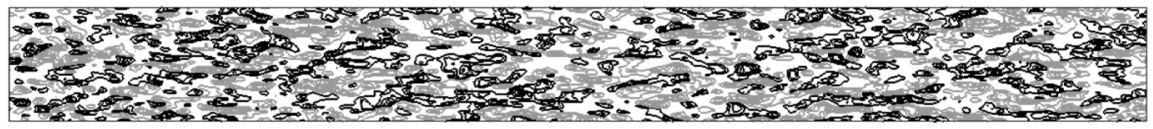

(f)

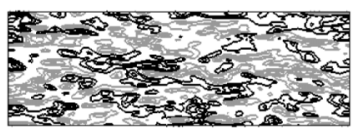

(g)

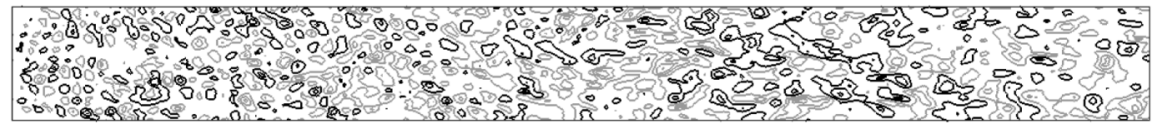

(h)

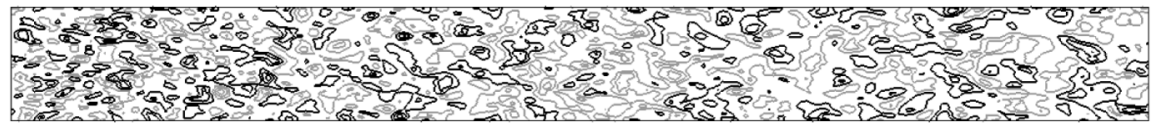

(i)

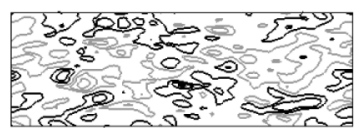

FIG. 14. Horizontal slices showing fluctuating streamwise velocity at ((a)-(c)) $y / h=-0.99$; ((d)-(f)) $y / h=-0.8$; $((\mathrm{g})-(\mathrm{i})) y / h=0$. ((a), (d), (g)): forced LES, $\tau_{v}=\tau_{r}=0.5 h / U_{b}$ (case 2); ((b), (e), (h)): forced LES, $\tau_{v}=\tau_{r}=0.1 h / U_{b}$ (case 3); ((c), (f), (i)): periodic LES. The contours correspond to $\pm 0.06,0.12,0.18,0.24,0.3$ for ((a)-(f)) graphs and $\pm 0.03,0.0725,0.115,0.1575,0.2$ for $((\mathrm{g})-(\mathrm{i}))$ graphs, with black contours for positive values and gray contours for negative values.

and a better reproduction is obtained by increasing this relaxation parameter, in comparison with $\tau_{r}$ (case 4). The reason for this influence lies in the fact that the resolved mean velocity is evaluated during the computation using a Gaussian temporal filter, with a large but finite filter width. Therefore, even in permanent state, the resolved mean velocity $\left\langle\widetilde{u_{i}}\right\rangle$ in Eq. (37) is not constant but oscillates at low frequency, such that the mean component $B_{i}$ of the forcing term oscillates as well, with an amplitude depending on the relaxation time $\tau_{v}$. However, Fig. 15 shows that the agreement of the long-time averaged velocity with the target profile $\left\langle\widetilde{u}_{i}\right\rangle^{\dagger}$ is still very satisfactory when the $\tau_{v}$ parameter is increased to $\tau_{v}=5 \mathrm{~h} / U_{b}$.

Since $\tau_{r}$ imposes the time scale of the ALF turbulent production, the question arises of making this parameter variable within the domain, as a function of the integral time scale, or eddy turnover time, $k / \varepsilon$,

$$
\tau_{r}=C_{r} \frac{k^{\dagger}}{\varepsilon^{\dagger}} .
$$

In the RANS/LES framework, this time scale can be computed from the RANS solution. Figure 16 shows an a priori test performed in order to calibrate the $C_{r}$ constant from the DNS data of Moser 

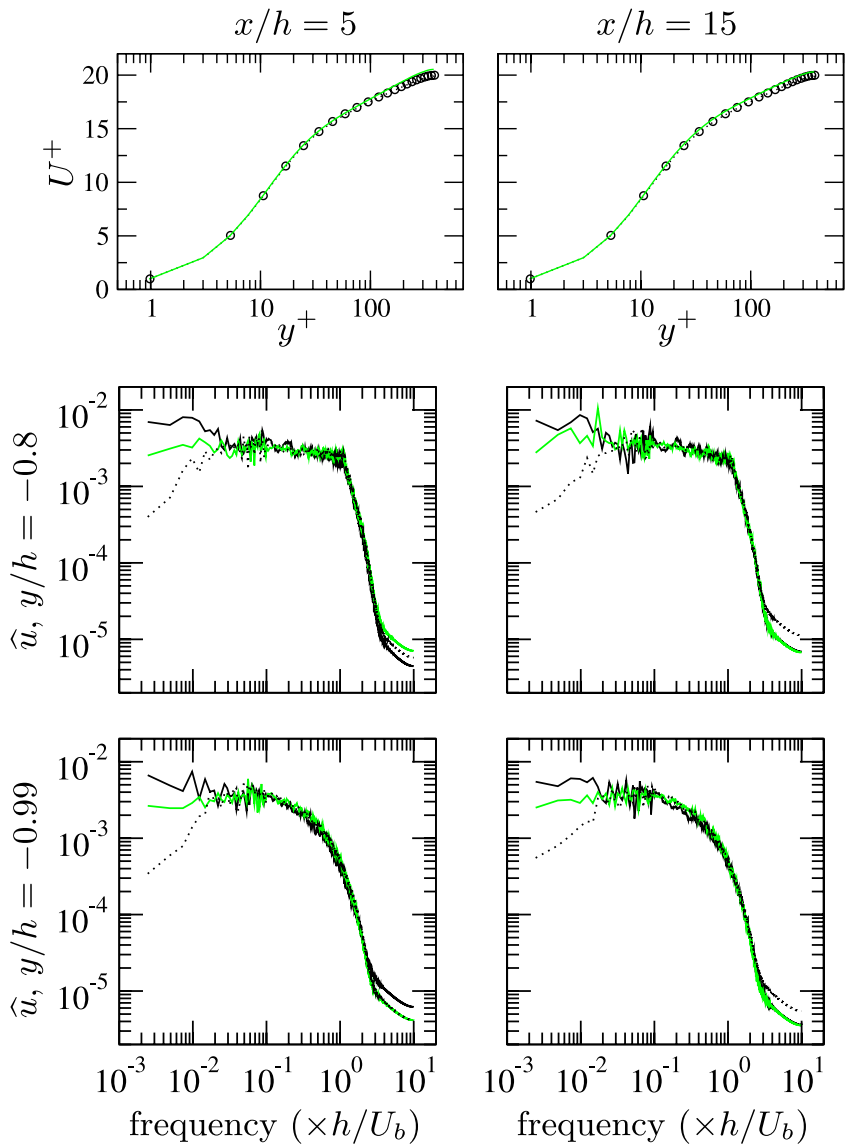

FIG. 15. Mean velocity (top) and temporal Fourier transform of streamwise velocity, at $y / h=-0.8$ (middle) and $y / h=$ -0.99 (bottom). Profiles at $x / h=5$ (left) and $x / h=15$ (right). $\circ$, black solid line: periodic LES; dotted lines: forced LES, $\tau_{r}=0.1 h / U_{b}$ and $\tau_{v}=0.1 h / U_{b}$ (case 3); green solid line: forced LES, $\tau_{r}=0.1 h / U_{b}$ and $\tau_{v}=5 h / U_{b}$ (case 4).

et al..$^{32}$ It can be seen that, in order to obtain a spatially variable $\tau_{r}$ of the same order of magnitude than the constant value $0.1 h / U_{b}$ that was successfully applied above, the coefficient $C_{r}$ must be as small as $C_{r}=0.01$. Taking into account the additional constraint that $\tau_{r}$ must remain larger than the time step $\Delta t$, the proposed formulation for $\tau_{r}$ is

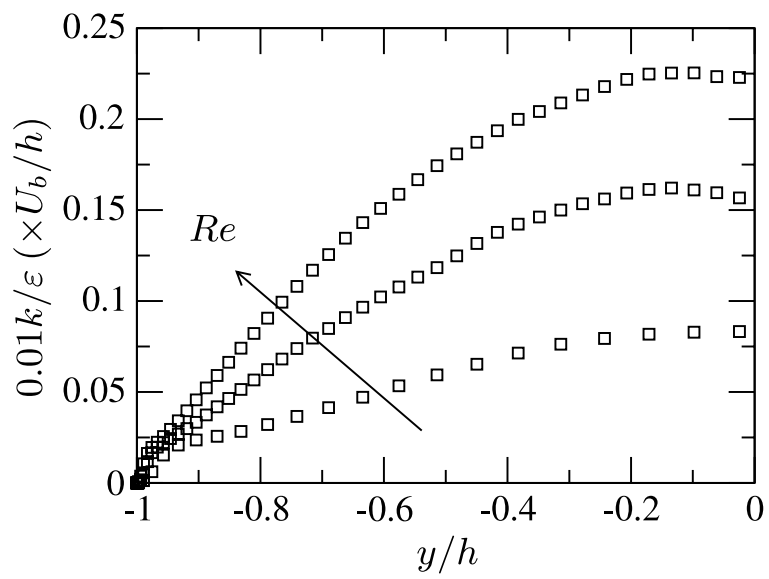

FIG. 16. Integral time scale (multiplied by $C_{r}=0.01$ ) in a channel flow at $R e_{\tau}=180,395,590$. DNS data of Moser et al. $^{32}$ 


$$
\tau_{r}=\max \left\{2 \Delta t, 0.01 \frac{k^{\dagger}}{\varepsilon^{\dagger}}\right\} .
$$

The results of the application of this spatially variable formulation for $\tau_{r}$, associated with $\tau_{v}=$ $5 h / U_{b}$, are shown in Fig. 17 (case 5). In order to circumvent the issue of the evaluation of the total turbulent energy $k^{\dagger}$ and the dissipation rate $\varepsilon^{\dagger}$ from resolved variables of the periodic LES, and anticipating the fact that, for applications in the hybrid RANS/LES context, these quantities will be provided by the RANS computation, Eq. (71) is simply computed here from the DNS data of Moser et al. ${ }^{32}$ at $R e_{b}=6881 \simeq 7000$. It can be seen in Fig. 17 that the forcing is satisfactory with this formulation of $\tau_{r}$, from both the statistical and the spectral point of view. Actually, the local nature of $\tau_{r}$ has a minor influence in this case and the results obtained are very similar to those of case 4 . It is however believed that the formulation in Eq. (71) is more general and, in particular, able to adjust the intensity of the forcing to the turbulence intensity of the flow, as computed by the RANS method. Consequently, this variable formulation is applied in the hybrid RANS/LES computations of Sec. IV B.

\section{Influence of the explicit averaging operator}

Through Eqs. (30) and (31), the ALF can be seen as a restoring force tending to drive the mean velocity and the resolved stresses toward target values. The method is therefore dependent on the approximation used to evaluate the first and second moments of the LES during the computation (as mentioned at the beginning of the present section, the computations presented above employed an exponential time filtering $\mathcal{G}_{T}$, Eq. (9), with the temporal filter width $T=100 h / U_{b}$ ). In particular, it was shown in Sec. IV A 2 that, since the approximate mean velocity $\mathcal{G}_{T}\left(\widetilde{u}_{i}\right)$ is subject to low-frequency oscillations, of the order of magnitude of $T$, the mean force $\left\langle f_{i}\right\rangle$ does not only influence the mean velocity but also the low-frequency turbulent fluctuations.

In Fig. 18, in order to investigate the effect of the approximate statistical averaging operator, an exponential filtering of reduced size $T=10 h / U_{b}$ (case 6a) and an exponential filtering of size $T=100 h / U_{b}$ associated with spatial averaging in the homogeneous direction $z$ (case $6 \mathrm{~b}$ ) are considered. The Fourier transforms are plotted close to the channel outlet in order to guarantee that the forced LES is fully developed. It is observed that, with $T=10 \mathrm{~h} / U_{b}$, the low frequency components of the velocity are significantly damped in the core of the flow $(y / h=0)$. In the vicinity of the wall $(y / h=-0.99)$, the velocity spectrum is also modified, with an overestimation at high frequencies.
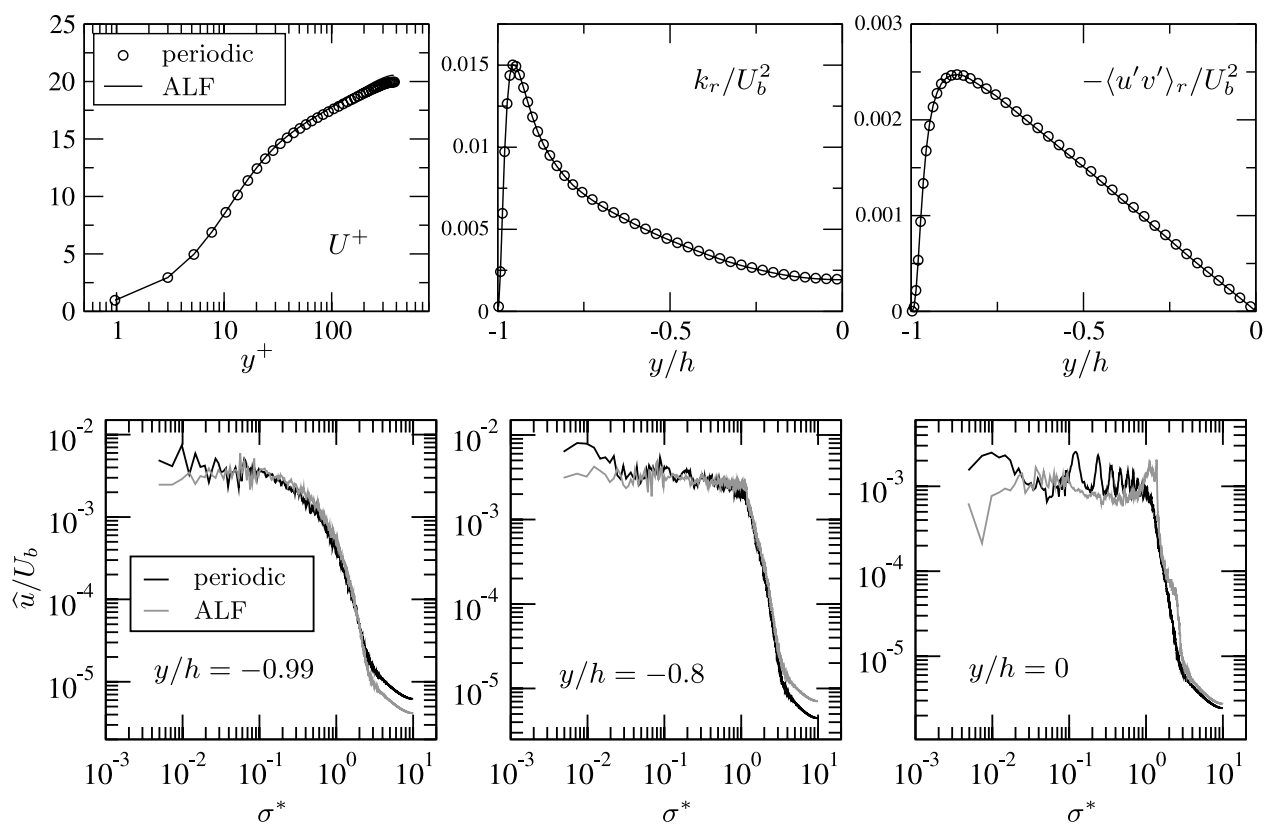

FIG. 17. Forced LES $\tau_{v}=5 h / U_{b}$ and $\tau_{r}=0.01 k / \varepsilon$ (case 5). Top: Mean velocity, turbulent energy, and turbulent shear stress. Bottom: Fourier transform of streamwise velocity for several distances to the wall. 

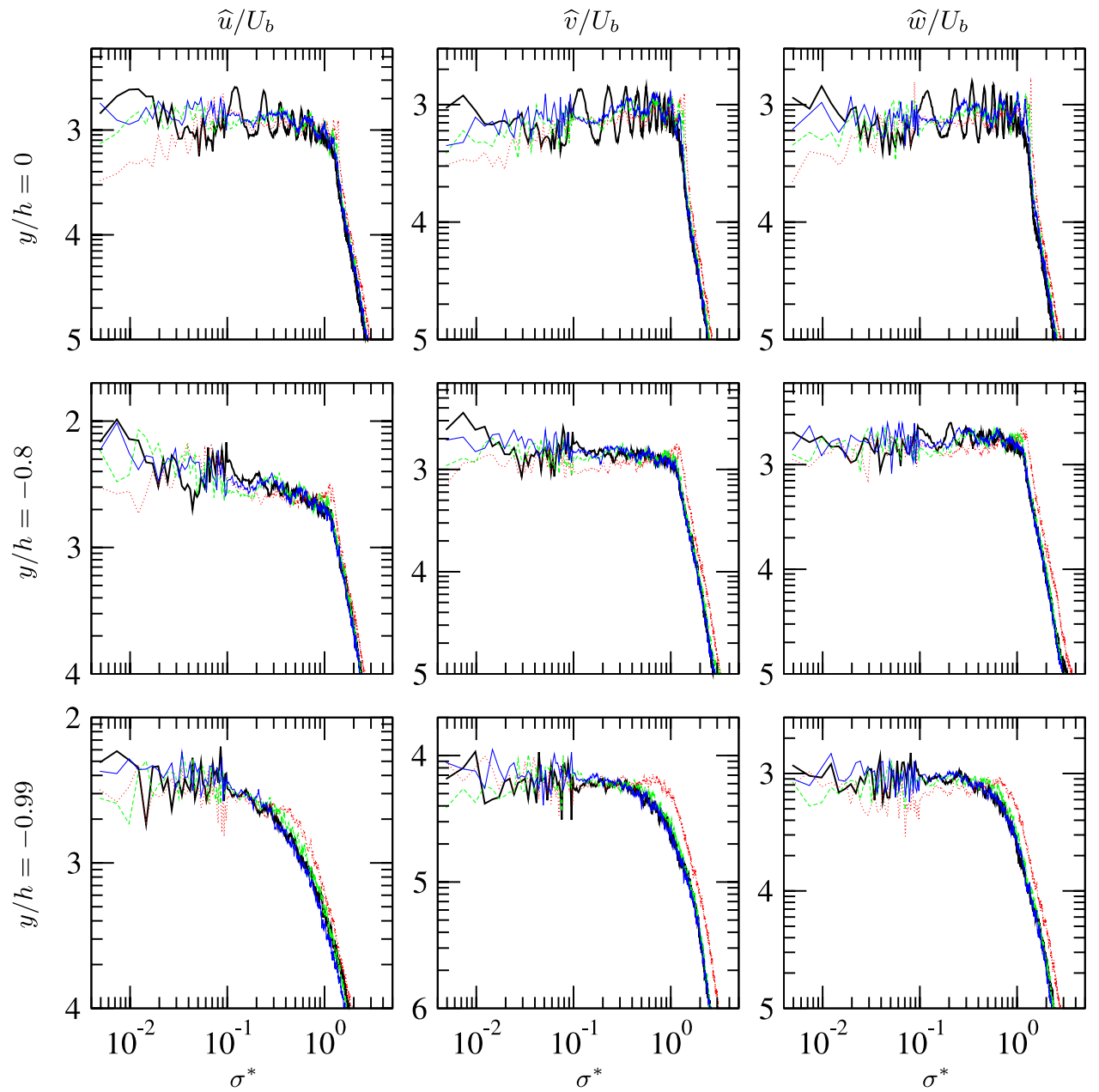

FIG. 18. Fourier transform of the three velocity components at $x / h=30$ and at several distances to the wall $(y / h=$ $0,-0.8,-0.99)$. Influence of the explicit averaging operator. Red dots: exponential filtering of size $T=10 h / U_{b}$ (case $\left.6 a\right)$; green dashes: exponential filtering of size $T=100 \mathrm{~h} / U_{b}$ (case 5); blue solid line: exponential filtering of size $T=100 \mathrm{~h} / U_{b}$ and spatial averaging in the $z$ direction; thick black solid line: periodic LES.

In contrast, the more accurate approximation of the statistical operator applied for case $6 \mathrm{~b}$ provides velocity spectra almost identical to those of the fully developed, unforced LES. However, since averaging in homogeneous directions is not possible in more complex geometries, for the sake of generality, the exponential filter with $T=100 h / U_{b}$ is considered a good compromise and is adopted for the hybrid RANS/LES computations of Sec. IV B.

To conclude the parametric study of the present section, the components of the mean force and the $A_{i j}$ tensor are plotted in Figs. 19 and 20, respectively, for the case 5 of Table II. It is observed that, as the mean velocity approaches the target value, the mean force rapidly decreases downstream of the inlet, but does not vanish, in particular close to the walls. As regards the $A_{i j}$ tensor, Fig. 20 reveals that the dominant components are $A_{11}$ and $A_{22}$, which, in particular, contribute to the shear stress budget, via the term $\left(A_{11}+A_{22}\right)\left\langle u^{\prime} v^{\prime}\right\rangle$. Similar to the mean force, $A_{11}$ and $A_{22}$ decrease in the streamwise direction of the channel but do not go to zero.

\section{B. Application to synthetic turbulence generation in zonal RANS/LES modeling}

Using the insight gained in Sec. IV A into the influence of the different ingredients of the ALF on the spatial development of the resolved fluctuations, the method is now applied to the zonal hybrid RANS/LES coupling. For the purpose of evaluating the performance of the ALF, results are compared 


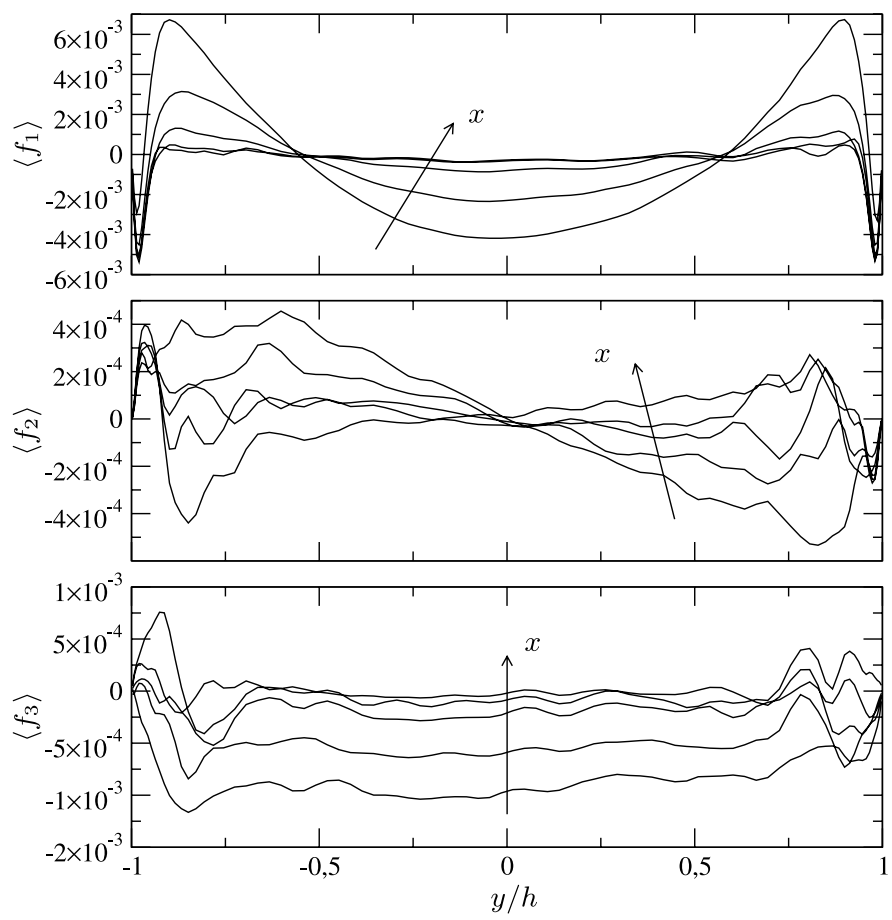

FIG. 19. Mean ALF components at $x / h=2,5,10,15,30$ (case 5).

to a well established method of generation of turbulent fluctuations at the inlet of the LES domain, the synthetic eddy method (SEM).

\section{Formulation of the coupling}

Channel flow RANS and LES solutions are computed in separate domains. The LES domain is located downstream of the RANS domain, with an overlap region. Since, in the present case, the
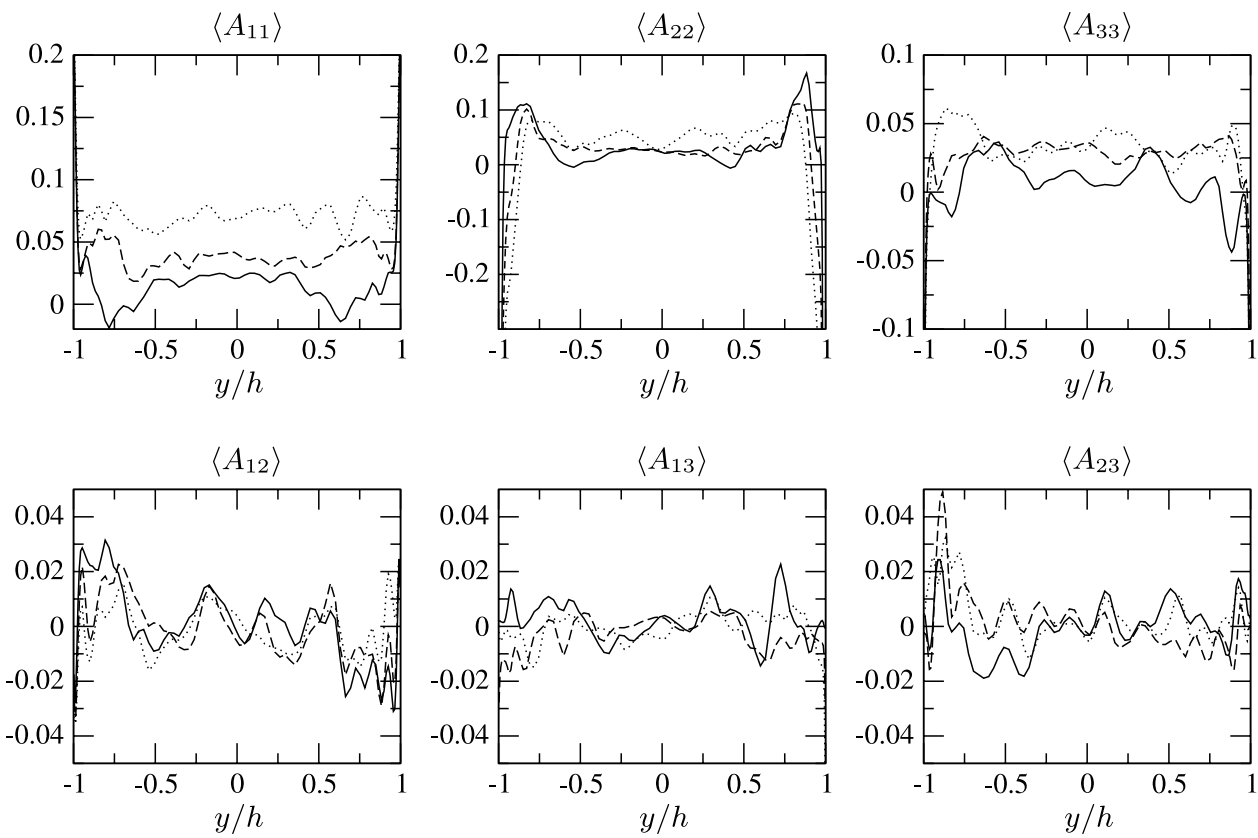

FIG. 20. Mean $A_{i j}$ components for case 5 at $x / h=5$ (dotted lines), 15 (dashed lines), and 30 (black solid line). 
LES computation does not influence the RANS computation (one-way coupling), periodic boundary conditions are used in RANS, in order to generate a fully developed solution. In Eqs. (35) and (37) that determine the $A_{i j}$ and $B_{i}$ coefficients of the ALF, the target mean velocity and resolved Reynolds stresses are

$$
\begin{aligned}
\left\langle\widetilde{u}_{i}\right\rangle^{\dagger} & =\left\langle u_{i}\right\rangle^{\text {RANS }}, \\
\left\langle u_{i}^{\prime} u_{j}^{\prime}\right\rangle^{\dagger} & =\left\langle u_{i}^{\prime} u_{j}^{\prime}\right\rangle^{\text {RANS }}-\left\langle\tau_{i j}\right\rangle,
\end{aligned}
$$

where the superscript RANS is the self-explanatory and $\left\langle\tau_{i j}\right\rangle$ is the contribution of the subgrid scales to the Reynolds stresses in the LES computation. Note that, in the RANS context, $\left\langle u_{i}^{\prime} u_{j}^{\prime}\right\rangle^{\mathrm{RANS}}$ denotes the Reynolds stress tensor.

The evaluation of $\left\langle\tau_{i j}\right\rangle$ is not straightforward since, for standard algebraic subgrid scale models, such as the Smagorinsky model used herein, only the deviatoric part $\left\langle\tau_{i j}^{d}\right\rangle$ of the subgrid scale tensor is modeled. To circumvent this limitation, $\left\langle\tau_{i j}\right\rangle$ is decomposed into

$$
\left\langle\tau_{i j}\right\rangle=\left\langle\tau_{i j}^{d}\right\rangle+\frac{2}{3} k_{\tau} \delta_{i j},
$$

where $k_{\tau}=\left\langle\tau_{i i}\right\rangle / 2$. In contrast to $\left\langle\tau_{i j}^{d}\right\rangle$ that is evaluated during the computation from the resolved fluctuations, the subgrid scale kinetic energy is modeled as

$$
k_{\tau}=C \frac{3 C_{K}}{2}\left(\frac{\varepsilon^{\mathrm{RANS}}}{\kappa_{c}}\right)^{2 / 3}, \quad C_{K}=1.5, C=0.35,
$$

which corresponds to the integration of a Kolmogorov spectrum beyond the cut-off wavenumber $\kappa_{c}=\pi /(\Delta x \Delta y \Delta z)^{1 / 3}$, with $C$ a calibration constant introduced to account for the fact that the implicit LES filter is not a spectral cut-off filter. This constant is calibrated based on the DNS data of Moser et $a .^{32}$ at $R e_{b}=6881$ and the reference periodic LES at $R e_{b}=7000$ of Sec. IV A, in order to ensure that the constraint

$$
\int_{0}^{h} k \mathrm{~d} y=\int_{0}^{h}\left(k_{r}+k_{\tau}\right) \mathrm{d} y
$$

is fulfilled, where $k$ and $\varepsilon$ are evaluated from the DNS, and $k_{r}$ and $\kappa_{c}$ from the LES.

According to the parametric study of Sec. IV A, $\tau_{v}=5 h / U_{b}$ and $\tau_{r}$ are evaluated from the RANS computation as

$$
\tau_{r}=\max \left(2 \Delta t, 0.01 \frac{k^{\mathrm{RANS}}}{\varepsilon^{\mathrm{RANS}}}\right),
$$

and the statistical averaging operator $\langle\cdot\rangle$ is approximated by an exponential time filter of temporal width $T=100 h / U_{b}$, using $\left\langle u_{i}\right\rangle^{\text {RANS }}$ and $\left\langle u_{i}^{\prime} u_{j}^{\prime}\right\rangle^{\text {RANS }}$ as initial values.

\section{Results}

The ALF method is applied to the same flow configuration as in Sec. IV A, with two major differences: the target statistics are not given by a periodic LES, but rather by a RANS computation, accounting for the subgrid scale contribution (Eq. (73)); only a restricted region, downstream of the inlet, is forced, using the local RANS statistics. The length of this overlapping region in the streamwise direction is denoted by $L_{x}^{f}$. The ALF is applied over the entire extent of the domain in the $y$ and $z$ directions.

In the RANS domain, a low-Reynolds number second moment closure, the EB-RSM ${ }^{21,33,34}$ is used. In the LES domain, of size $20 \pi h \times 2 h \times \pi h$, the Smagorinsky model is used and the mesh is similar to the one used in Sec. IV, with near-wall cells of size $\left(\Delta x^{+}, \Delta y_{\min }^{+}, \Delta z^{+}\right) \simeq(50,2,15)$. At the inlet of the LES domain, the mean velocity $\left\langle u_{i}\right\rangle^{\text {RANS }}$ of the RANS computation is imposed without any superimposed fluctuations. 
The spatial development of the flow in the LES domain is observed by plotting the streamwise evolution of the friction coefficient,

$$
C_{f}=\frac{u_{\tau}^{2}}{\frac{1}{2} U_{b}^{2}}
$$

and the error functions

$$
e_{k_{r}}=\frac{\int_{-h}^{h}\left(k_{r}-k_{r}^{*}\right)}{\int_{-h}^{h} k_{r}^{*}}, \quad e_{\left\langle u^{\prime} v^{\prime}\right\rangle}=\frac{\int_{-h}^{h}\left(\left|\left\langle u^{\prime} v^{\prime}\right\rangle\right|-\left|\left\langle u^{\prime} v^{\prime}\right\rangle^{*}\right|\right)}{\int_{-h}^{h}\left|\left\langle u^{\prime} v^{\prime}\right\rangle^{*}\right|},
$$

where the asterisk denotes the statistics of the fully developed flow, obtained from the periodic simulation presented in Sec. IV. Several lengths $L_{x}^{f}$ of the forcing area are considered in Fig. 21. For each simulation, the origin is shifted to coincide with the end of the forcing region, $\widetilde{x}=x-L_{x}^{f}$. It can be seen that, independently of the forcing length $L_{x}^{f}$, the turbulent fluctuations generated by the ALF in the overlapping region are sustained downstream. However, when $L_{x}^{f}$ is increased, the spectral content of the flow improves at the end of the forcing region (not shown here), and as a consequence, the transient decrease of the fluctuations just downstream of the forcing region is reduced. A forcing length of $L_{x}^{f}=5 h$ is sufficient to obtain a very satisfactory development of the flow in the LES domain. With this forcing length, the maximum overestimation of the friction coefficient is within $6 \%-7 \%$ of the fully developed value $C_{f}^{*}$ and is observed just after the end of the forcing region $(\widetilde{x} \simeq h)$. Downstream $\widetilde{x}=5 h$, the friction coefficient remains within $\pm 2.5 \%$ of $C_{f}^{*}$. The error functions $e_{k_{r}}$ and $e_{\left\langle u^{\prime} v^{\prime}\right\rangle}$ for the resolved turbulent energy and shear stress, respectively, are below $15 \%$ in absolute value throughout the domain.

The forcing length $L_{x}^{f} / h=5$ is now selected for comparison with a hybrid RANS/LES computation using the Synthetic Eddy Method (SEM) of Jarrin et al. ${ }^{9}$ at the LES inlet boundary. In this method, the domain do not overlap, and unsteady Dirichlet boundary conditions for the velocity at
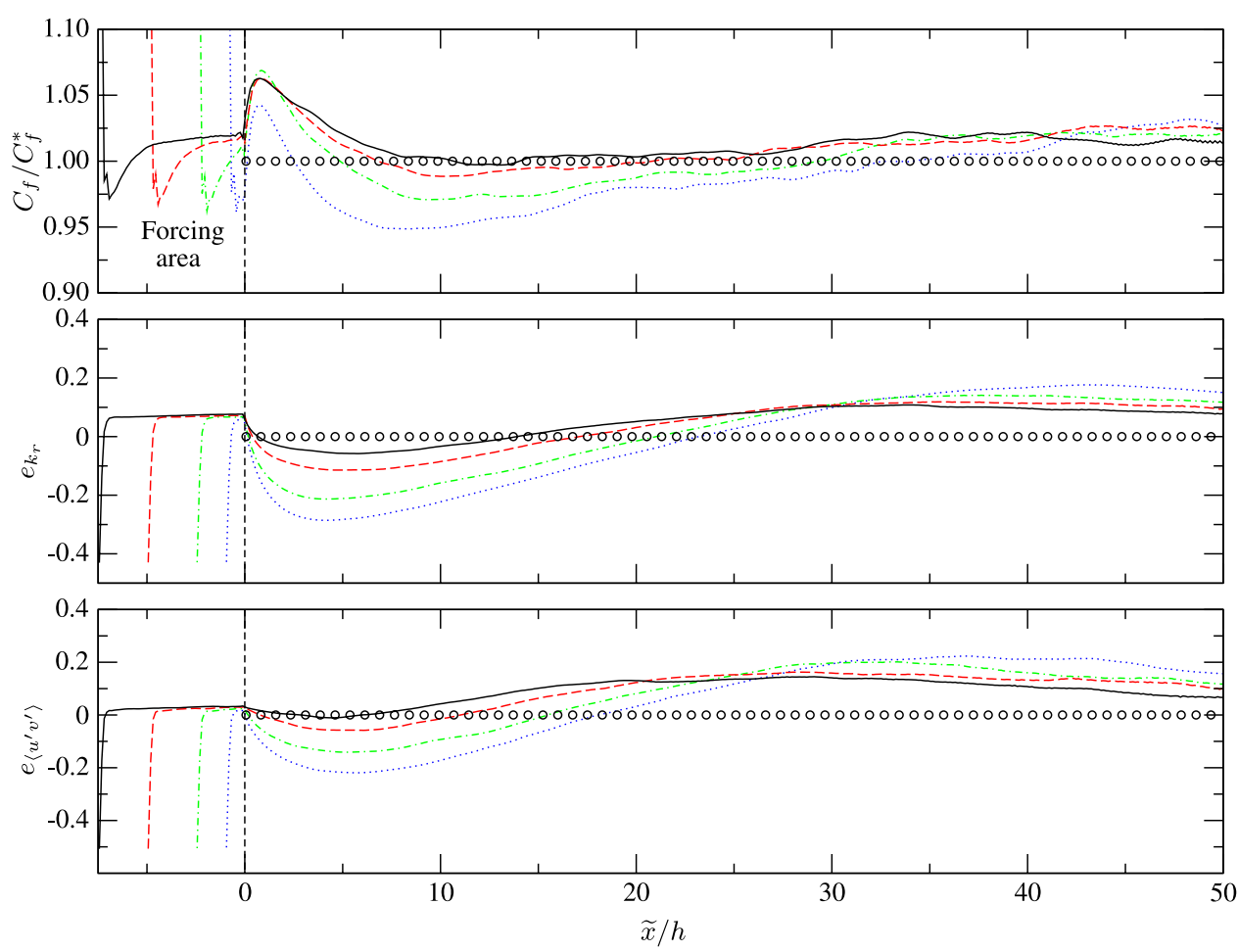

FIG. 21. Evolution of the friction coefficient and the error functions $e_{k_{r}}$ and $e_{\left\langle u^{\prime} v^{\prime}\right\rangle}$. Symbols: periodic LES; lines: results in the LES domain of the hybrid RANS/LES computation using the ALF for $L_{x}^{f} / h=1$ (blue dotted lines), 2.5 (red dashed-dotted lines), 5 (red dashed lines), 7.5 (black solid line). 
the inlet of the LES domain are generated using the superposition of several coherent synthetic eddies evolving in a virtual "box" surrounding the inlet plane, using the RANS statistics as input parameters. Applying the renormalization procedure of Lund, ${ }^{10}$ the SEM generates velocities in the inlet plane that satisfy the first- and second-order moments of the RANS computation. This method was validated by Jarrin $\mathrm{et} \mathrm{al.}{ }^{7}$ in the context of RANS to LES coupling with a two-equations eddy-viscosity model. As described in Appendix B, the present SEM method is reformulated in order to take advantage of the anisotropic prediction of the Reynolds stress tensor provided by the EB-RSM second moment closure in the RANS region.

Figure 22 compares the friction coefficient and the error functions defined by Eqs. (78) and (79) given by the two approaches. For the sake of completeness, the results obtained using the isotropic version of the forcing method (ILF) are plotted as well. In contrast with Fig. 21, the profiles are plotted as a function of the original $x$ coordinates such that $x / h=5$ corresponds to the end of the forcing region for the ALF and the ILF computations. Globally, the discrepancies with the fully developed solution are substantially smaller with the forcing method (ALF or ILF) than with the SEM. However, the complete convergence toward the developed solution is longer with the forcing: at the end of the LES domain, the flow statistics are closer to the fully developed, periodic LES solution with the SEM than with the ALF or the ILF. Moreover, comparing the ILF and ALF results, it can been seen that the development of the solution downstream of the forcing region is only slightly slowed down when the anisotropy of the Reynolds stress is not enforced. These results show that the length of the forcing region is sufficient for the anisotropy to naturally develop as soon as the level of energy is imposed. However, it can be observed, in particular at the beginning of the forcing region, that the shear stress much more rapidly reaches the correct level with the ALF, due to the fact that it is specifically forced. This result shows that the ALF is preferable to the ILF, in order to avoid a stress depletion in the forcing region that can be at the origin of grid-induced separation. ${ }^{35}$
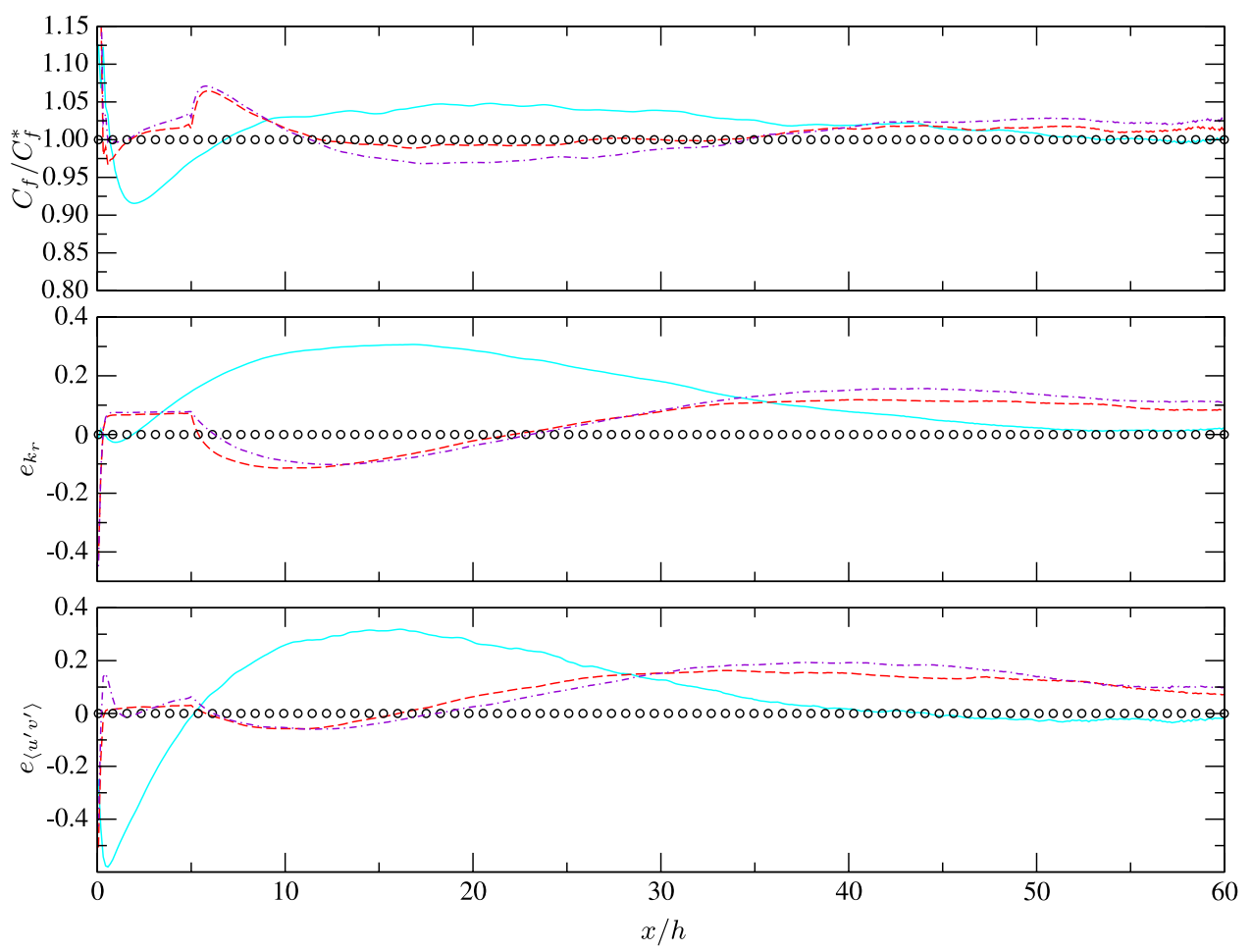

FIG. 22. Evolution of the friction coefficient and the error functions $e_{k_{r}}$ and $e_{\left\langle u^{\prime} v^{\prime}\right\rangle}$ downstream of the LES inlet. Hybrid RANS/LES computation using the ALF with $L_{x}^{f} / h=5$ (red dashed lines); the ILF with $L_{x}^{f} / h=5$ (purple dashed-dotted lines); the SEM (cyan solid line) compared with periodic LES (o). 

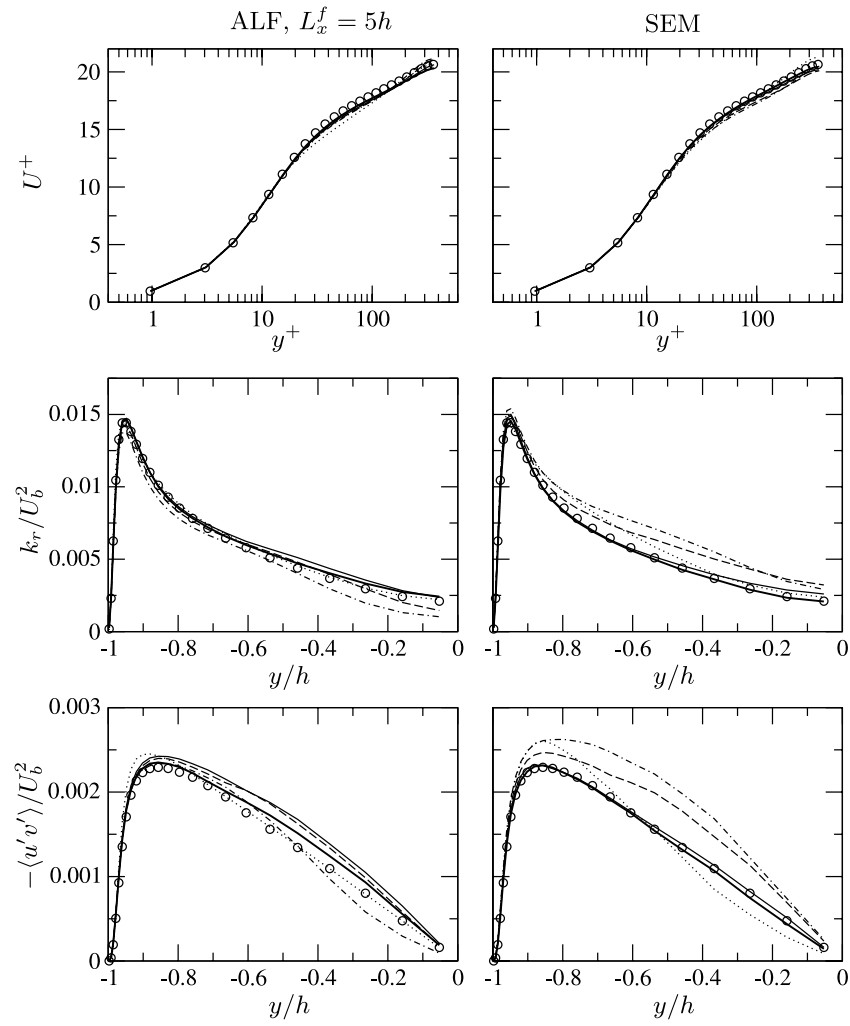

FIG. 23. Mean velocity, turbulent kinetic energy, and turbulent shear stress at $x / h=5$ (dotted lines), 15 (dashed-dotted lines), 25 (dashed lines), 40 (black solid line, thin line), 60 (black solid line, thick line). Hybrid RANS/LES computation using the ALF with $L_{x}^{f} / h=5$ (left) or SEM (right). Symbols: periodic LES; lines: hybrid computations.

Figure 23 gives a more local view of preceding observations. The mean velocity, the resolved kinetic energy, and the resolved shear stress profiles are plotted at various streamwise locations. With the ALF, the mean velocity and the Reynolds stress profiles are always close to those of the periodic computation. In particular, at $x / h=5$, which corresponds to the end of the forcing region, the agreement is very satisfactory. This is mainly due to the accurate EB-RSM predictions provided to the ALF as target statistics, since the contribution of the subgrid scales, taken into account using Eqs. (73)-(75), is not significant in this case. Downstream of the forcing region, the turbulent stresses are first moderately damped in the core of the channel, which is visible at $x / h=15$, associated with an overestimation of the maximum velocity. Downstream, the missing turbulent fluctuations build up, leading to an overestimation of the turbulent stresses in the core region of the flow, and eventually a convergence towards the developed values. Concerning the SEM, the statistics of the flow approach their fully developed values around $x / h=40$, but significant discrepancies are observed upstream of this location.

Some remarks concerning the computational cost can be made from Table III where the average CPU time per time step and the average number of sub-iterations necessary to reach convergence in the pressure correction step are summarized. As expected, increasing the forcing length tends to increase the CPU time of the computation. The global number of additional floating point operations necessary to compute the parameters $A_{i j}$ and $B_{i j}$ of the ALF is proportional to $N_{f}$. Although there are large uncertainties in CPU time measurements, the evaluated coefficient of proportionality is about 1.5, i.e., a forcing in $10 \%$ of the computational domain leads to an extra CPU cost of $15 \%$. More noticeable is the fact that the number of sub-iterations in the pressure correction step of the predictor-corrector algorithm is virtually unchanged by the introduction of the forcing. The situation is completely different in case of the SEM. Indeed, with this method-as for other synthetic turbulent inflow methods such as that of Batten et al. ${ }^{8}$ for instance-the velocity imposed at the inlet does not 
TABLE III. Comparison of the cost of the different computations. $N_{f} / N$ : ratio of the number of forced cells to the total number of cells; CPU: average CPU time per time step; $n_{\Delta p}$ : average number of sub-iterations for the pressure correction step. (The CPU time of the periodic LES is not given because the computation domain is smaller.)

\begin{tabular}{lcccc}
\hline \hline$L_{x}^{f} / h$ & $N_{f} / N(\%)$ & CPU (s) & $n_{\Delta p}$ \\
\hline \multicolumn{5}{c}{ ALF } \\
\hline 1 & 1.6 & & 1.77 & 82.3 \\
2.5 & 4 & 1.84 & 81.8 \\
5 & 8 & 1.91 & 81.3 \\
7.5 & 11.9 & & 1.91 & 81.1 \\
& & SEM & & 802.6 \\
$\ldots$ & $\ldots$ & & 7.24 & \\
& & Periodic LES & & 81.5 \\
\hline$\ldots$ & $\ldots$ & & $\ldots$ & \\
\hline \hline
\end{tabular}

satisfy the divergence-free constraint. The projection of velocity onto the solenoidal plane yields a significant computational over-cost and unphysical pressure fluctuations close to the inlet (see Poletto et $a l^{36}{ }^{36}$. In contrast, with the ALF, the mean velocity imposed at the inlet is divergence-free and turbulent fluctuations are generated inside the domain by a forcing term. Consequently, although the transient at the beginning of the simulation is slightly longer with the ALF due to the relaxation time introduced by the Gaussian averaging, the total simulation cost is three times lower with the ALF that with the SEM in this case. This moderate CPU cost, in comparison with the SEM or similar methods, is a significant asset of the ALF.

\section{CONCLUSIONS}

A new forcing method has been proposed for the hybrid RANS/LES modeling of turbulence, in order to enrich the RANS solution at the interface between a upstream RANS and a downstream LES region. The method, the so-called Anisotropic Linear Forcing (ALF), introduces a time-dependent term in the momentum equation that acts as a restoring force toward a target statistical state. The very generic form of the forcing, a tensorial linear function of filtered velocity, is such that the ALF can be seen as a generalization of several body forces for RANS/LES volume coupling proposed in the literature. ${ }^{12,13,15}$ In addition, for isotropic turbulence, the ALF reduces to the isotropic linear forcing proposed by Lundgren ${ }^{19}$ and further analyzed by Rosales and Meneveau. ${ }^{20}$

After verifying that the interesting properties of linear forcing of isotropic turbulence are preserved with the ALF formulation, it was shown in homogeneous turbulence that the ALF is able to enforce any turbulent anisotropy, within an accuracy that is controlled by the relaxation time scale $\tau_{r}$.

In order to investigate in detail the influence of the parameters of the ALF, the case of a spatially developing channel flow at $R e_{b}=7000$ has been considered, where the ALF is used to drive the flow towards the fully developed statistics of an auxiliary periodic computation. This case showed the ability of the ALF to rapidly generate coherent turbulent fluctuations, despite inlet boundary conditions independent of time. The sensitivity study is used to parametrize the method for subsequent zonal RANS/LES applications. The selected parameters are such that the first and second velocity moments of the forced LES are very close to the target statistics of the ALF and a realistic spectral content of the flow is ensured. The main parameter is the eddy turnover time that can be easily computed from a RANS simulation.

Applications of the ALF in zonal hybrid RANS/LES simulations of the developing channel flow were presented. A second moment closure, the EB-RSM ${ }^{21}$ was used in the RANS region. The parameters and target statistics of the ALF are evaluated from the RANS computation and the forcing is applied in a region of overlapping of the RANS and LES domains. The inflow boundary condition 
for the LES is simply the mean velocity obtained in the RANS domain. A short forcing length of 5 channel half-width is sufficient to obtain a satisfactory spatial development of the flow, with minor adjustments downstream of the forced region. Although the distance after the inlet necessary to reach the developed level of the friction coefficient is still significant, this original approach for zonal RANS to LES one-way coupling improves the results obtained using the synthetic eddy method at the LES inlet-a RANS/LES coupling method first proposed by Jarrin et al. ${ }^{7}$ Moreover, the additional CPU time due to the method used to generate turbulent fluctuations is significantly smaller with the ALF than with the SEM.

The results presented in the present article are very encouraging and open the possibility for the ALF method to be applied in various situations. Although the present article focuses on zonal RANS/LES approaches, with discontinuous interfaces, the method has the potential for being applied in global RANS/LES approaches, with diffuse interfaces. In contrast with other approaches, such as those presented above, it does not require any information about the direction of the flow: since the forcing term is evaluated from local information only, it is of very general application. In particular, the method can be easily applied to damp the time-dependent fluctuations at the outlet of the LES region in case of a LES to RANS diffuse interface, and can be potentially applied in a tangential RANS/LES coupling as well, such that it may be a all-in-one solution for embedded LES. Furthermore, if experimental data are substituted for target statistics from a RANS computation, the ALF can be used to impose realistic "inlet" conditions (specifically, to generate a realistic flow field at the end of the forcing region) to a LES or DNS computation. Finally, future work will be dedicated to the extension of the method to the volume forcing of transported scalar fields, and to the promotion of turbulent fluctuations in the region of transition from a RANS to a LES behavior (the so-called gray zone) in global, or continuous, hybrid RANS/LES methods.

\section{ACKNOWLEDGMENTS}

Part of the work was carried out using the HPC resources of GENCI (Grand Equipement National de Calcul Intensif) under the allocation 2014-2a0912.

\section{APPENDIX A: FRAME INVARIANCE OF THE ANISOTROPIC LINEAR FORCING}

As described in Sec. II C, the ALF is defined as

$$
\mathbf{f}=\mathbf{A}(\widetilde{\mathbf{u}}-\langle\widetilde{\mathbf{u}}\rangle)+\frac{1}{\tau_{v}}\left(\langle\mathbf{u}\rangle^{\dagger}-\langle\widetilde{\mathbf{u}}\rangle\right) .
$$

$\mathbf{A}$ is the solution of

$$
\mathbf{A} \mathbf{R}+\mathbf{R A}=\frac{1}{\tau}\left(\mathbf{R}^{\dagger}-\mathbf{R}\right)
$$

where $\mathbf{R}$ denotes the resolved stress tensor and $\mathbf{R}^{\dagger}$ the target resolved stress tensor. The explicit expression for $\mathbf{A}$ is given by Eq. (35).

A general change of reference frame is described by an Euclidean transformation

$$
\begin{aligned}
\mathbf{x}^{\star} & =\mathbf{Q}(t) \mathbf{x}+\mathbf{b}(t), \\
t^{\star} & =t+\theta .
\end{aligned}
$$

$\mathbf{Q}$ is an orthogonal matrix satisfying $\mathbf{Q} \mathbf{Q}^{T}=\mathbf{Q}^{T} \mathbf{Q}$, where the superscript $T$ denotes the transposition. By definition, the ALF term is frame-invariant, or objective, if and only if

$$
\mathbf{f}^{\star}=\mathbf{Q f} .
$$

A few assumptions are necessary for this property to be satisfied.

(i) The LES filter is isotropic. This assumption is made by Speziale 37,38 to show that

$$
\widetilde{\mathbf{u}}=\mathbf{Q}^{T}\left(\widetilde{\mathbf{u}}^{\star}+\mathbf{\Omega} \wedge \mathbf{x}^{\star}\right),
$$


with $\Omega$ the rotation vector of the reference frame, that is the dual vector of the skew-symmetric matrix $\mathbf{Q} \dot{\mathbf{Q}}^{T}$,

$$
Q_{i k} \dot{Q}_{j k}=\epsilon_{i m j} \Omega_{m}
$$

( $\epsilon_{i m j}$ denotes the permutation tensor). This assumption is necessary to ensure that the resolved stress tensor $\mathbf{R}$ is objective. Indeed, the application of the Reynolds operator to Eq. (A5) gives

$$
\langle\widetilde{\mathbf{u}}\rangle=\mathbf{Q}^{T}\left(\langle\widetilde{\mathbf{u}}\rangle^{\star}+\boldsymbol{\Omega} \wedge \mathbf{x}^{\star}\right)
$$

such that the filtered velocity fluctuation is objective,

$$
(\widetilde{\mathbf{u}}-\langle\widetilde{\mathbf{u}}\rangle)=\mathbf{Q}^{T}\left(\widetilde{\mathbf{u}}^{\star}-\left\langle\widetilde{\mathbf{u}}^{\star}\right\rangle\right),
$$

and consequently, the resolved stress tensor $\mathbf{R}$ is objective as well,

$$
\mathbf{R}^{\star}=\mathbf{Q R}^{T} .
$$

(ii) The target statistics of the ALF exhibit the same transformation properties as the resolved statistics, that is,

$$
\begin{aligned}
\langle\mathbf{u}\rangle^{\dagger} & =\mathbf{Q}^{T}\left(\left(\langle\mathbf{u}\rangle^{\dagger}\right)^{\star}+\mathbf{\Omega} \wedge \mathbf{x}^{\star}\right), \\
\mathbf{R}^{\dagger} & =\mathbf{Q}^{T}\left(\mathbf{R}^{\dagger}\right)^{\star} \mathbf{Q} .
\end{aligned}
$$

This assumption is not restrictive, since they are typically given by an experiment, a DNS, or a RANS computation. RANS models are formulated in such a way that the modeled Reynolds stress satisfies Eq. (A10).

(iii) The $\tau_{v}$ and $\tau_{r}$ parameters of the forcing are objective scalars,

$$
\tau_{v}=\tau_{v}^{\star}, \quad \tau_{r}=\tau_{r}^{\star} .
$$

This assumption is satisfied if they are imposed constant or function of objective quantities, such as the eddy turnover time in the present article.

Now that the framework is specified, it is a simple matter to check that the mean part of the ALF-the second term in the rhs of Eq. (A1)—is objective, by combining Eqs. (A6), (A9), and (A11). As regards the fluctuating part, Eq. (A7) shows that the fluctuating filtered velocity is objective, such that the last property to demonstrate is the objectivity of the tensor A. Explicit expression (35) symbolically writes

$$
\mathbf{A}=g(\mathbf{R}, \mathbf{H}),
$$

where $\mathbf{H}$, defined by

$$
\mathbf{H}=\frac{1}{\tau_{r}}\left(\mathbf{R}^{\dagger}-\mathbf{R}\right),
$$

is an objective tensor (see Eqs. (A8), (A10), and (A11)),

$$
\mathbf{H}^{\star}=\mathbf{Q H} \mathbf{Q}^{T} .
$$

Moreover, the $g$ function in Eq. (A12) is an isotropic tensor function, ${ }^{39}$

$$
g\left(\mathbf{Q R} \mathbf{Q}^{T}, \mathbf{Q H} \mathbf{Q}^{T}\right)=\mathbf{Q} g(\mathbf{R}, \mathbf{H}) \mathbf{Q}^{T} .
$$

The combination of Eqs. (A8), (A13), and (A14) shows that A is an objective tensor, and consequently, the ALF term is objective under assumptions (i)-(iii).

\section{APPENDIX B: FORMULATION OF THE SYNTHETIC EDDY METHOD AND COUPLING WITH A SECOND MOMENT CLOSURE}

With the synthetic eddy method of Jarrin et al., ${ }^{9}$ unsteady Dirichlet boundary conditions for the velocity are generated using a set of coherent synthetic eddies evolving in a virtual "box" surrounding 
the inlet plane. The method uses the decomposition of Lund et al. ${ }^{10}$

$$
\tilde{u}_{i}=\left\langle\tilde{u}_{i}\right\rangle^{\dagger}+a_{i j}^{\dagger} X_{j}
$$

where the first term on the rhs is the target mean velocity at the inlet and the second term is the fluctuating part. $a_{i j}^{\dagger}$ is the lower triangular matrix defined by the Cholesky factorization of the target resolved stress tensor,

$$
\left\langle u_{i}^{\prime} u_{j}^{\prime}\right\rangle^{\dagger}=a_{i k}^{\dagger} a_{j k}^{\dagger},
$$

and $X_{j}$ is a centered and unit random variable. The Lund et al. ${ }^{10}$ decomposition ensures that the first and second order velocity moments are $\left\langle\widetilde{u}_{i}\right\rangle^{\dagger}$ and $\left\langle u_{i}^{\prime} u_{j}^{\prime}\right\rangle^{\dagger}$, respectively.

The specificity of the SEM, compared to other synthetic inflow methods as that of Batten et al., ${ }^{8}$ is the definition of the $X_{j}$ variable. It mathematically expresses the phenomenology of the method,

$$
X_{j}=\frac{1}{\sqrt{N}} \sum_{\lambda=1}^{N} \epsilon_{j}^{\lambda} f_{\sigma(\mathbf{x})}\left(\mathbf{x}-\mathbf{x}^{\lambda}\right) .
$$

$\mathbf{x}^{\lambda}$ is the position of the eddy $\lambda$ in the virtual box $\mathcal{B}$. It is initially randomized, with a uniform probability density, and then advanced in time during the computation by

$$
\mathbf{x}^{\lambda}(t+\Delta t)=\mathbf{x}^{\lambda}(t)+U_{b} \Delta t
$$

where $U_{b}$ is the bulk velocity at the inlet. $\epsilon_{j}^{\lambda}$ and $f_{\sigma}$ give the sign and the amplitude of the contribution of the eddy $\lambda$ to the fluctuating field: $\epsilon_{j}^{\lambda}$ is randomized within the set $\{-1,1\}$ and the shape function of the eddies $f_{\sigma}$, which gives coherence to the velocity at the inlet, is given by

$$
f_{\sigma(\mathbf{x})}\left(\mathbf{x}-\mathbf{x}^{\lambda}\right)=\sqrt{\frac{|\mathcal{B}|}{\sigma^{3}}} f\left(\frac{x-x^{\lambda}}{\sigma}\right) f\left(\frac{y-y^{\lambda}}{\sigma}\right) f\left(\frac{z-z^{\lambda}}{\sigma}\right),
$$

with

$$
f(x)=\left\{\begin{array}{cc}
\sqrt{\frac{3}{2}}(1-|x|) & \text { if }|x|<1, \\
0 & \text { elsewhere. }
\end{array}\right.
$$

It can be seen that the shape function $f_{\sigma}$ is parametrized by a characteristic length scale $\sigma$ of the eddies. The proper definition of $\sigma$ is an important feature of the SEM, as confirmed by the study of Pamiès et al. ${ }^{14}$ Note that the box $\mathcal{B}$ containing the eddies

$$
\mathcal{B}=\prod_{i=1}^{3}\left[\min _{\mathbf{x} \in \mathcal{S}}\left(x_{i}-\sigma(\mathbf{x})\right), \max _{\mathbf{x} \in \mathcal{S}}\left(x_{i}+\sigma(\mathbf{x})\right)\right]
$$

also depends on $\sigma$.

The SEM can be easily applied to the one-way coupling of a LES inlet with a RANS computation, by using

$$
\begin{aligned}
\left\langle\widetilde{u_{i}}\right\rangle^{\dagger} & =\left\langle u_{i}\right\rangle^{\text {RANS }}, \\
\left\langle u_{i}^{\prime} u_{j}^{\prime}\right\rangle^{\dagger} & =\left\langle u_{i}^{\prime} u_{j}^{\prime}\right\rangle^{\text {RANS }}
\end{aligned}
$$

in Eq. (B1). The development of the flow in the LES region is sensitive to the definition of the characteristic length scale $\sigma$. In order to evaluate $\sigma$ from the $k-\omega$ SST eddy-viscosity model used as the RANS model, ${ }^{40}$ Jarrin et al. ${ }^{7}$ proposed the relation

$$
\sigma=\min \left\{\max \left\{\frac{k^{\mathrm{RANS}^{3 / 2}}}{\varepsilon^{\mathrm{RANS}}}, \max \{\Delta x, \Delta y, \Delta z\}\right\}, C_{\kappa} \delta\right\},
$$

where $C_{K}=0.41$ is the Kármán constant and $\delta$ is the boundary layer thickness.

In order to exploit the information about the turbulent anisotropy provided by a second moment RANS closure, three different length scales $\sigma_{i}$, corresponding to the three directions $x_{i}$, can be 
substituted for the single characteristic length scale $\sigma$, leading to the anisotropic shape function

$$
f_{\sigma(\mathbf{x})}\left(\mathbf{x}-\mathbf{x}^{\lambda}\right)=\prod_{i=1}^{3} \sqrt{\frac{\left|\mathcal{B}_{i}\right|}{\sigma_{i}}} f\left(\frac{x_{i}-x_{i}^{\lambda}}{\sigma_{i}}\right),
$$

and the anisotropic virtual box

$$
\mathcal{B}=\prod_{i=1}^{3} \mathcal{B}_{i}, \quad \text { with } \quad \mathcal{B}_{i}=\left[\min _{\mathbf{x} \in \mathcal{S}} x_{i}-\sigma_{i}, \max _{\mathbf{x} \in \mathcal{S}} x_{i}+\sigma_{i}\right] .
$$

The proposed anisotropic length scales $\sigma_{i}$ is a straightforward generalization of the expression of Jarrin et al., ${ }^{7}$

$$
\sigma_{i}=\min \left\{\max \left\{\frac{\left(\frac{3}{2}\left\langle u_{i}^{\prime 2}\right\rangle^{\mathrm{RANS}}\right)^{3 / 2}}{\varepsilon^{\mathrm{RANS}}}, \Delta_{i}\right\}, C_{\kappa} \delta\right\}
$$

In this relation, the isotropic upper bound is the same as in Eq. (B8), in order to avoid unphysical length scales in the center of the channel.

Figure 24 presents the streamwise evolution in the LES region of the friction coefficient $C_{f}$ and error functions (79) for the channel flow at $R e_{b}=7000$ with the same mesh as in Sec. IV A. In order to investigate the influence of the accurate prediction of the turbulent anisotropy by the RANS model, a linear eddy-viscosity model, the $k-\omega$ SST model, is used in addition to the second moment EB-RSM closure. With the EB-RSM, both the isotropic, Eq. (B8), and anisotropic, Eq. (B11), definitions of the length scale are applied for comparison.

It is observed that when $\sigma$ is isotropic, the use of the second moment closure EB-RSM in the RANS region slightly degrades the development of the flow in the LES region compared to the simulation using the SST model in the RANS region. This is somewhat surprising given that the EB-RSM
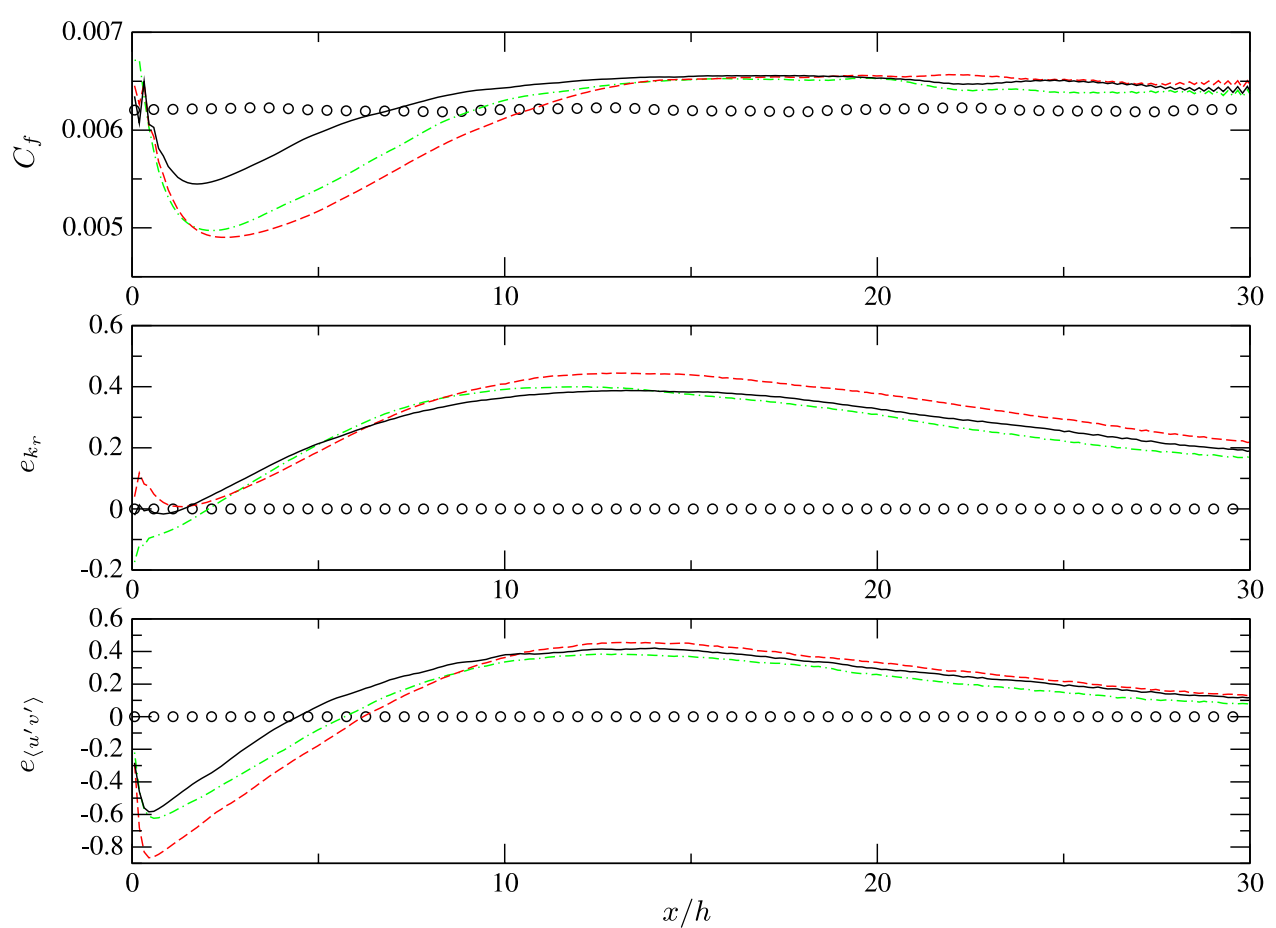

FIG. 24. Evolution of the friction coefficient and the error functions $e_{k_{r}}$ and $e_{\left\langle u^{\prime} v^{\prime}\right\rangle}$ (Eq. (79)) downstream the LES inlet. Influence of the prediction of the anisotropy: green dashed-dotted lines: SST/Smagorinsky coupling; red dashed lines: EB-RSM/Smagorinsky coupling, with the isotropic length scale $\sigma$; black solid line: EB-RSM/Smagorinsky coupling, with the anisotropic length scales $\sigma_{i}$. 
predicts flow statistics in closer agreement with those of the periodic LES than the SST model. Nevertheless, it can be inferred that the eddy-viscosity hypothesis of the SST model, giving $\left\langle v^{\prime 2}\right\rangle=2 k / 3$, tends to overestimate $\left\langle v^{\prime 2}\right\rangle$ and thus to promote the shear stress production $P_{12}=-\left\langle v^{\prime 2}\right\rangle \partial\langle u\rangle / \partial y$ downstream of the LES inlet, resulting in a better development of the flow. Using an anisotropic length scale in association with the EB-RSM, Fig. 24 shows that the spatial development of the flow in the LES region is significantly improved.

${ }^{1}$ P. R. Spalart, W.-H. Jou, M. K. Strelets, and S. R. Allmaras, "Comments of feasibility of LES for wings, and on a hybrid RANS/LES approach," in Advances in DNS/LES, Proceedings of the First AFOSR International Conference on DNS/LES, 4-8 August, Ruston, LA, edited by C. Liu and Z. Liu (Greyden Press, Columbus, OH, USA, 1997).

${ }^{2}$ F. Menter, M. Kuntz, and R. Bender, "A scale-adaptive simulation model for turbulent flow predictions," in Proceedings of the 41st AIAA Aerospace Sciences Meeting and Exhibit, AIAA paper 2003-0767, Reno, Nevada, 2003.

${ }^{3}$ A. Fadai-Ghotbi, C. Friess, R. Manceau, and J. Borée, "A seamless hybrid RANS-LES model based on transport equations for the subgrid stresses and elliptic blending," Phys. Fluids 22, 055104 (2010).

${ }^{4}$ P. Sagaut, S. Deck, and M. Terracol, Multiscale and Multiresolution Approaches in Turbulence (Imperial College Press, 2006).

5 A. Keating, U. Piomelli, and E. Balaras, "A priori and a posteriori tests of inflow conditions for large-eddy simulation," Phys. Fluids 16(12), 777-788 (2004).

${ }^{6}$ A. Keating, G. De Prisco, and U. Piomelli, "Interface conditions for hybrid RANS/LES calculations,” Int. J. Heat Fluid Flow 27, 777-788 (2006).

${ }^{7}$ N. Jarrin, R. Prosser, J. C. Uribe, S. Benhamadouche, and D. Laurence, "Reconstruction of turbulent fluctuations for hybrid RANS/LES simulations using a synthetic-eddy Method,” Int. J. Heat Fluid Flow 30, 435-442 (2009).

${ }^{8}$ P. Batten, U. Goldberg, and S. Chakravarthy, “Interfacing statistical turbulence closures with large-eddy simulation," AIAA J. 42, 485-492 (2004).

${ }^{9}$ N. Jarrin, S. Benhamadouche, D. Laurence, and R. Prosser, "A synthetic-eddy-method for generating inflow conditions for large-eddy simulations," Int. J. Heat Fluid Flow 27, 585-593 (2006).

${ }^{10}$ T. S. Lund, X. Wu, and K. D. Squires, "Generation of turbulent inflow data for spatially-developing boundary layer simulations," J. Comput. Phys. 140, 233-258 (1998).

${ }^{11}$ H. Xiao and P. Jenny, "A consistent dual-mesh framework for hybrid LES/RANS modeling," J. Comput. Phys. 231, 1848-1865 (2012).

${ }^{12}$ A. Spille-Kohoff and H. J. Kaltenbach, "Generation of turbulent inflow data with a prescribed shear-stress profile," in DNS/LES Progress and Challenges, Proceedings of the 3rd AFOSR International Conference on DNS/LES, Arlington, TX, USA, edited by C. Liu, L. Sakell, and T. Beutner (Greyden Press, Columbus, OH, USA, 2001).

${ }^{13}$ R. Laraufie, S. Deck, and P. Sagaut, "A dynamic forcing method for unsteady turbulent inflow conditions," J. Comput. Phys. 230(23), 8647-8663 (2011).

${ }^{14}$ M. Pamiès, P.-E. Weiss, E. Garnier, S. Deck, and P. Sagaut, "Generation of synthetic turbulent inflow data for large eddy simulation of spatially evolving wall-bounded flows," Phys. Fluids 21(4), 045103 (2009).

15 J. U. Schlüter, H. Pitsch, and P. Moin, "Outflow conditions for integrated large eddy simulation/Reynolds-averaged Navier-Stokes simulations," AIAA J. 43(1), 156-164 (2005).

${ }^{16}$ D. A. Von Terzi and J. Fröhlich, "Segregated coupling of large-eddy simulations with downstream Reynolds-averaged Navier-Stokes calculations," Comput. Fluids 39, 1314-1331 (2010).

${ }^{17}$ Y. Benarafa, O. Cioni, F. Ducros, and P. Sagaut, "RANS/LES coupling for unsteady turbulent flow simulation at high Reynolds number on coarse meshes," Comput. Methods Appl. Mech. Eng. 195, 2939-2960 (2006).

${ }^{18}$ E. Labourasse and P. Sagaut, "Reconstruction of turbulent fluctuations using a hybrid RANS/LES approach," J. Comput. Phys. 182, 301-336 (2002).

${ }^{19}$ T. S. Lundgren, "Linearly forced isotropic turbulence," Center of Turbulence Research Annual Research Briefs 123-176 (2003).

${ }^{20}$ C. Rosales and C. Meneveau, "Linear forcing in numerical simulations of isotropic turbulence: Physical space implementations and convergence properties," Phys. Fluids 17(9), 095106 (2005).

${ }^{21}$ R. Manceau and K. Hanjalić, "Elliptic blending model: A new near-wall Reynolds-stress turbulence closure," Phys. Fluids 14(2), 744-754 (2002).

${ }^{22}$ H. K. Vertseeg and W. Malalasekera, An introduction to Computational Fluid Dynamics: The Finite Volume Method (Longman Scientific and Technical, 1995).

${ }^{23}$ C. D. Pruett, T. B. Gatski, C. E. Grosch, and W. D. Thacker, "The temporally filtered Navier-Stokes equations: Properties of the residual stress," Phys. Fluids 15(8), 2127 (2003).

${ }^{24}$ M. Scheidler, "The tensor equation $A X+X A=\Phi(A, H)$, with applications to kinematics of continua," J. Elasticity $36(2)$, $117-153$ (1993).

${ }^{25}$ L. Rosati, "A novel approach to the solution of the tensor equation $A X+X A=H$," Int. J. Solids Struct. 37(25), 3457-3477 (2000).

${ }^{26}$ A. Hoger and D. E. Carlson, "On the derivative of the square root of a tensor and Guo's rate theorems," J. Elasticity 14(3), 329-336 (1984).

${ }^{27}$ A. A. Wray, "A selection of test cases for the validation of large-eddy simulations of turbulent flows," AGARD Advisory Report 345, 1998, Chapter: HOM02 Decaying Isotropic Turbulence.

${ }^{28}$ M. Germano, "Differential filters for the large eddy numerical simulation of turbulent flows," Phys. Fluids 29, 1755-1766 (1986).

${ }^{29}$ S. Bose, P. Moin, and F. Ham, "Explicitly filtered large eddy simulation on unstructured grids," Center of Turbulence Research Annual Briefs 87-96 (2011). 
${ }^{30}$ J. L. Lumley and G. R. Newman, “The return to isotropy of homogeneous turbulence,” J. Fluid Mech. 82, 161-178 (1977).

31 J. L. Lumley, "Computational modeling of turbulent flows," Adv. Appl. Mech. 18, 123-176 (1978).

32 R. D. Moser, J. Kim, and N. N. Mansour, "Direct numerical simulation of turbulent channel flow up to $R e_{\tau}=590$," Phys. Fluids 11(4), 943-945 (1999).

${ }^{33}$ A. Fadai-Ghotbi, Ch. Friess, R. Manceau, T. B. Gatski, and J. Borée, "Temporal filtering: A consistent formalism for seamless hybrid RANS-LES modeling in inhomogeneous turbulence,” Int. J. Heat Fluid Flow 31, 378-389 (2010).

${ }^{34}$ F. Dehoux, Y. Lecqoq, S. Benhamadouche, R. Manceau, and L.-E. Brizzi, "Algebraic modeling of the turbulent heat fluxes using the elliptic blending approach-Application to forced and mixed convection regimes," Flow, Turbul. Combust. 88(1), 77-100 (2012).

35 P. R. Spalart, "Detached-eddy simulation,” Annu. Rev. Fluid Mech. 41, 181-202 (2009).

${ }^{36}$ R. Poletto, T. Craft, and A. Revell, "A new divergence free synthetic eddy method for the reproduction of inlet flow conditions for embedded LES," Flow, Turbul. Combust. 91(3), 519-539 (2013).

${ }^{37}$ C. G. Speziale, "Subgrid scale stress models for the large eddy simulation of rotating turbulent flows," Geophys. Astrophys. Fluid Dyn. 33, 199-222 (1985).

${ }^{38}$ I. Luca and A. Sadiki, "New insight into the functional dependence rules in turbulence modelling," Int. J. Eng. Sci. 46(11), 1053-1062 (2008).

${ }^{39}$ G. F. Smith, "On isotropic functions of symmetric tensors, skew-symmetric tensors and vectors," Int. J. Eng. Sci. 9, 899 (1971).

${ }^{40}$ F. Menter, "Two-equation eddy-viscosity turbulence models for engineering applications," AIAA J. 32(8), 1598-1605 (1994). 